\title{
Novel organo-modifier for thermally-stable polymer-layered silicate nanocomposites
}

\author{
N.Tz. Dintcheva ${ }^{1,2}$, S. Al-Malaika ${ }^{2}$, E. Morici ${ }^{1}$ \\ (1) Dipartimento di Ingegneria Civile, Ambientale, Aerospaziale, dei Materiali, Università di \\ Palermo, Viale delle Scienze, Ed. 6, 90128 Palermo, Italy \\ (2) Polymer Processing and Performance Research Unit, School of Engineering and Applied \\ Science, Aston University, Aston Triangle, Birmingham, B4 7ET, UK
}

\begin{abstract}
A new novel approach for the stabilisation of polymer-clay nanocomposites has been investigated based on reacting chemically an antioxidant function, a hindered phenol moiety, with an organic modifier based on a quaternary ammonium salt. The chemically linked antioxidant-containing organic modifier (AO-OM) was then introduced into natural montmorillonite (MMt) through a cation-exchange reaction resulting in antioxidantcontaining organo-modified clay (AO-OM-MMt). The new antioxidant-containing modified clay, along with other organo-modified clays having a similar organo-modifier but without the reacted antioxidant, were characterised by spectroscopic, thermogravimetric and x-ray diffraction techniques and tested for their thermo-oxidative stability.
\end{abstract}

PA11-based clay nanocomposites samples containing the AO-OM-MMt and the other organo-modified clays, both without and with an added (i.e. not chemically reacted) hindered phenol antioxidant (similar to the one used in the AO-OM) were prepared by melt processing and examined for their processing and long-term thermal-oxidative stability at high temperatures. It was shown that although the new organo-modifier, AO-OM, was also susceptible to the Hoffman elimination reaction, the nanocomposites containing this newly modified clay (PA11/AO-OM-MMt) showed higher melt processing and long-term thermo-oxidative stability, along with excellent clay dispersion and exfoliation, compared to the other PA11-nanocomposites examined here (with and without the conventionally added antioxidant). It is suggested here that the excellent overall performance observed for the PA11/AO-OM-MMt nanocomposites is due to an in-situ partial release of low molecular weight antioxidant species having stabilising functionalities that are capable of acting locally at the interface between the inorganic clay platelets and the polymeric matrix which is a critical area for the onset of degradation processes.

Keywords:Polymer-clay nanocomposites; PA11; Antioxidant-containing organomodifier; modified montmorillonites ${ }^{*}$ Corresponding author. Tel: +44 (0)121 204 3372. Fax: +44 121204 3679. E-mail address: s.al-malaika@aston.ac.uk (S. Al-Malaika). 


\section{Introduction}

Interest in the area of clay-reinforced polymer nanocomposites (PNCs) remains unabated due to their perceived benefits: the low nanofiller loading (1-10 wt\% compared to $30-50 \mathrm{wt} \%$ of microfiller in conventional composites) needed to achieve enhanced performance properties, the abundance of silicate minerals in nature and their low production cost. Although the primary interest in PNCs is related to their reinforcement aspect, they have several other beneficial properties such as flammability resistance, mechanical performance, electrical/electronic and barrier properties [1-5]. Silicates that have attracted most attention in PNC applications are based on natural minerals, particularly, the plate-like montmorillonite (MMt): platelets made up of octahedrally and tetrahedrally substituted layered silicates possessing negative charges balanced, normally, by alkali or alkali earth cations, located between the platelets or in the galleries, see Scheme 1 (a). To achieve PNCs with good performance, the silicate platelets must be well dispersed and delaminated in the matrix [5-9]. However, despite the proven benefits of polymer nanocomposites mentioned above, they are used today only in niche industrial applications mainly due to the challenges of the dispersion of nanoparticles in hydrocarbon polymer matrices during the melt processing and their inherently lower thermo- and photooxidative stability when compared to that of the neat matrix [10-26].

Different strategies have been implemented to achieving compatibility, dispersion and distribution of the silicates into the polymer matrices. A common practice is the introduction of an organo-modifier (OM) between the platelets of the silicate stacks [5-9]. The use of appropriate dispersing additives [27, 28] or selected copolymer structures [29] during melt processing had also been considered but the results achieved were not satisfactory because, although these agents help to improve the dispersion of the silicate stacks in the polymer matrix, exfoliation of the clay morphology was not achieved. Instead, the introduction of OM between the platelets of silicate stacks has been shown to help to promote both the dispersion and changes in the clay morphology. The introduction of the OM can be performed by ion exchange between the alkali or alkali earth cations, such as sodium ions located in the galleries, and the organic cations, such as quaternary ammonium, see Scheme 1 (b). Usually, the ammonium cations, with amphiphilic character, have different hydrocarbon tails and other organic groups, e.g. methyl, short alkyl chains with terminal $-\mathrm{OH}$, benzene. However, the main problem with using a quaternary ammonium $\mathrm{OM}$ is related to its thermal instability under nanocomposite production conditions, e.g., high processing temperatures and mechanical stress, which are required for polymer melt processing. Thermal degradation of the quaternary ammonium OM occurs via a Hoffman elimination reaction [10-13] resulting in the formation of acidic sites, free radicals and other reactive species, all of which are detrimental to the performance and long-term thermal stability of the PNCs $[14,15,21]$. Autocatalytic metal ions, e.g. iron, present in the clay structure [20] and the physical "loss" of stabilisers [1617], are further sources of instability of PNCs. Concerted efforts made to compensate for the overall oxidative instability of the final PNCs by blending in the best commercial antioxidant systems have not been highly 
successful. One of the main issues here concerns the "loss" of antioxidants and stabilisers from the nanocomposites through their entrapment and/or adsorption on the large clay surfaces.

Alternative routes to formulating thermally-stable clay intercalants have included the use of phosphonium salts [30] or organo-silicon compounds, e.g. POSS, a polyoligomeric silsesquioxane [31]; these too did not show much improvement in terms of long-term thermoxidative stability of the final PNC.

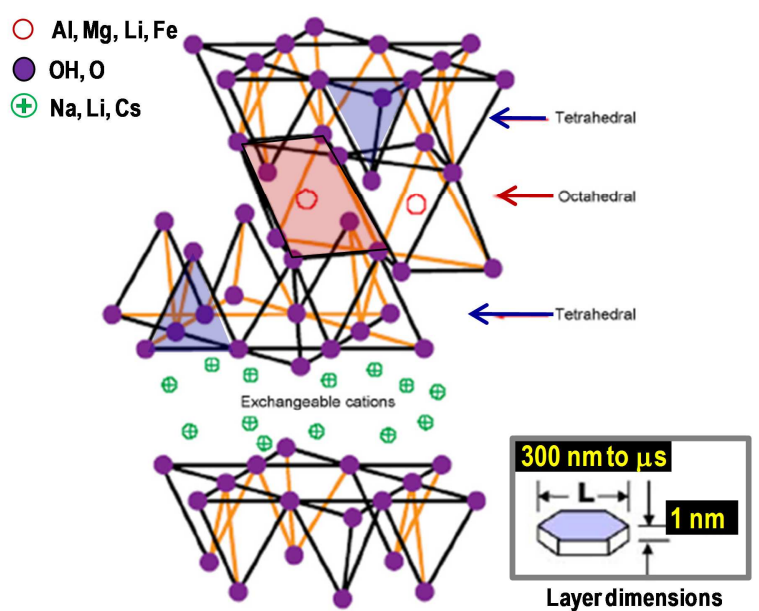

(a)

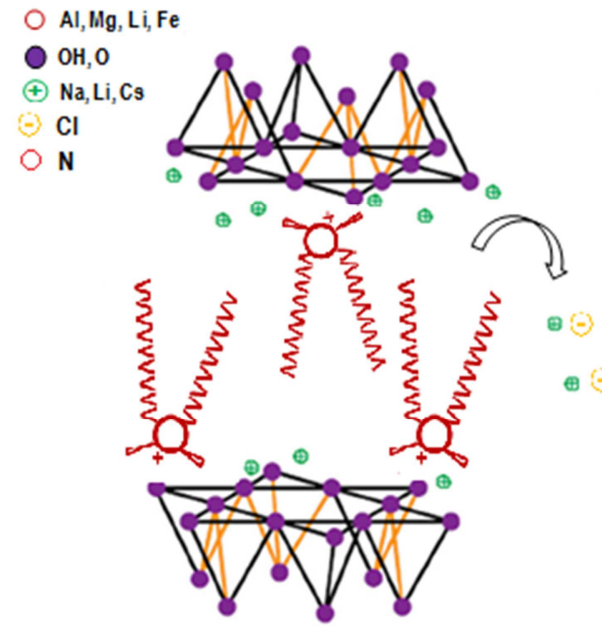

(b)

Scheme 1: Structure of layered silicates (a) [4] and intercalated silicates structures (b)

In this work, a novel organo-modifier was produced through chemical reaction of an antioxidant function (AO), such as a hindered phenol, with the ammonium quaternary salt (the clay organic modifier, OM), resulting in a chemically-linked AO-containing organo-modifier (AO-OM). This was followed by an ion exchange reaction with the natural MMt to produce a thermally stable organo-modified montrmorillonite (AO-OM-MMt) nanofiller which was compounded subsequently by melt mixing with the bio-based polyamide 11 (PA11).

The nanofiller dispersion and the morphology of the nanocomposites were assessed and the thermo-oxidative resistance of the AO-OM/MMt and that of the corresponding polyamide nanocomposite samples (PA11/AOOM-MMt) were examined. The stabilising action of AO-OM/MMt was compared with similar organically modified clays but without the antioxidant, and with similarly formulated PA11 nanocomposite but using a laboratory-made OM-MMt, containing the same organo-modifier used to formulate the new AO-OM, in the absence and presence of an added commercial hindered phenol antioxidant moiety similar to that used in the preparation of the new AO-OM. 


\section{Experimental}

\subsection{Materials}

The polyamide (PA) used in this work was a PA-11 (Nylon 11 from Sigma Aldrich), with glass transition temperature $T_{g}=46^{\circ} \mathrm{C}$, melting temperature $T_{m}=198^{\circ} \mathrm{C}$ and density $\rho=1.026 \mathrm{~g} / \mathrm{cm}^{3}$ at $25^{\circ} \mathrm{C}$. The nanofillers were ex. Southern Clay Products and their characteristics are given in Table 1. The organic modifier (OM) oleylmethylbis(2-hydroxyethyl) ammonium chloride, ETHOQUAD® O/12 PG $\left(\mathrm{C}_{23} \mathrm{H}_{48} \mathrm{NO}_{2}\right.$, molecular weight = $370.64 \mathrm{~g} / \mathrm{mol}$ ) obtained from Akzo Nobel® was used to prepare the antioxidant containing organo-modifier (AO-OM). A hindered phenol antioxidant (AO), octadecyl-3-(3, 5-di-tert-butyl-4-hydroxyphenyl), Irganox® $1076\left(\mathrm{C}_{35} \mathrm{H}_{62} \mathrm{O}_{3}\right.$, molecular weight $\left.=531 \mathrm{~g} / \mathrm{mol}\right)$, ex. Ciba ${ }^{\circ}$ Specialty Chemicals, was used to prepare the reactive-antioxidant moiety, $\mathrm{r}-\mathrm{AO}$. The nominal composition of all the PA11-based nanocomposites produced with modified and unmodified clays was: PA11/Clay at 95:5 wt\% (based on the inorganic content of the clay). One of the PA11-nanocomposite samples containing the organo-modified nanofiller was stabilised in a conventional way by adding to the formulation a large amount, i.e. $1 \mathrm{wt} \%$, of the hindered phenol antioxidant Irganox 1076; this was used for reference purposes and has the code PA11/OM-MMt/Irg.

Table 1: Commercial clay nanofillers used and their characteristics (manufacturer's data)

\begin{tabular}{|c|c|c|c|c|c|c|}
\hline Code & $\begin{array}{c}\text { layered silicate } \\
\text { nanofiller }\end{array}$ & Type & $\begin{array}{c}\text { Quat Surfactant } \\
\text { (organic modifier) }\end{array}$ & $\begin{array}{c}\text { Quat Conc } \\
\text { meq } / 100 \mathrm{~g} \text { clay }\end{array}$ & $\begin{array}{l}\mathrm{d}_{001} \\
\mathrm{~nm}\end{array}$ & $\begin{array}{c}\text { Specific } \\
\text { gravity } \\
\mathrm{g} / \mathrm{cm}^{3}\end{array}$ \\
\hline NaMMTt & Cloisite $® \mathrm{Na}^{+}$ & $\begin{array}{c}\text { natural } \\
\text { montmorillonite }\end{array}$ & None & 92.6 & 1.17 & 2.86 \\
\hline $\mathrm{CL}^{30 B}{ }^{(*)}$ & Cloisite $® 30 B$ & montmorillonite & $\begin{array}{c}\text { MT2EtOH: } \\
\text { methyl, tallow, bis-2- } \\
\text { hydroxyethyl, quaternary } \\
\text { ammonium } \\
\\
\mathrm{CH}_{2} \mathrm{CH}_{2} \mathrm{OH}\end{array}$ & 90 & 1.85 & 1.98 \\
\hline
\end{tabular}

Note: $\left.{ }^{*}\right)$ CL30B was considered for comparison with lab-made OM-MMt, see Supplementary Information.

\subsection{Preparation of the reactive Antioxidant ( $r-A O)$}

The preparation of the reactive (towards the organic modifier ETHOQUAD) antioxidant (Irganox-acid, r-AO) was performed according to reaction 1 as follows: a suspension of $2 \mathrm{~g}(3.77 \mathrm{mmol})$ of Irganox ${ }^{\circledR} 1076$ in a solution of methanol/water $(45 \mathrm{~mL}+15 \mathrm{~mL})$ and $0.65 \mathrm{~g}(12 \mathrm{mmol})$ sodium methoxide (MeONa) was refluxed under $\mathrm{N}_{2}$ atmosphere for 3 hours at $70{ }^{\circ} \mathrm{C}$, then cooled down and left at room temperature overnight. The resulting mixture was filtered to remove the side product (stearyl alcohol), the filtrate acidified with concentrated hydrochloric acid $(\mathrm{HCl})$ and filtered to obtain the reactive antioxidant in the form of a slightly yellow solid. The product was washed with water and dried at $70{ }^{\circ} \mathrm{C}$ overnight resulting in a white powder 
$(0.78 \mathrm{~g}$, yield $74 \%)$. The identity of the r-AO was confirmed by MS, ${ }^{1} \mathrm{H}$ and ${ }^{13} \mathrm{C}$ NMR and FTIR (see Supplementary Information Section S1).

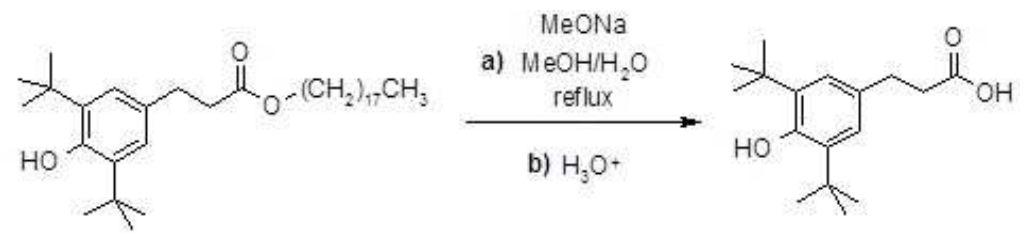

\subsection{Modification of the Organic Modifier (OM) to Antioxidant-containing OM (AO-OM)}

The modification of the commercial OM (ETHOQUAD® O/12 PG ) to AO-OM was performed according to reaction 2 as follows: a solution of ETHOQUAD® O/12 PG (2.45 mmol, $1.0 \mathrm{~g})$, Irganox acid (5 mmol, $1.4 \mathrm{~g})$ and N,N'-dicyclohexylcarbodiimide (DCC, $5 \mathrm{mmol}, 1.0 \mathrm{~g}$ ) in $20 \mathrm{~mL}$ dry THF was stirred under $\mathrm{N}_{2}$ atmosphere for 48 hours. The solution was then filtered to remove a white precipitate (dicyclohexylurea). The solvent was subsequently removed from the filtrate under reduced pressure leaving a solid product (AO-OM); full characterisation of this product will be discussed later (see results).

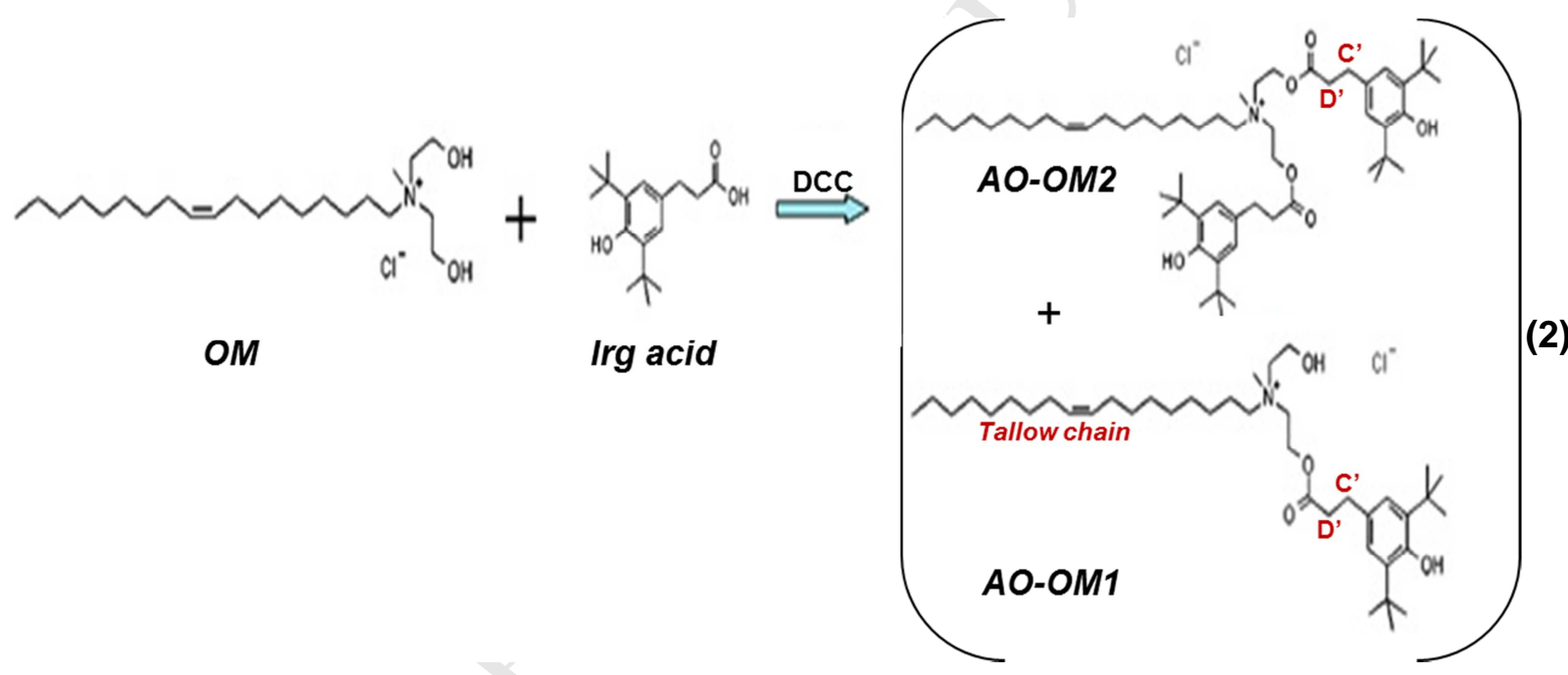

\subsection{Preparation of Montmorrilonite Clay-Containing the AO-OM (AO-OM-MMt)}

The antioxidant-containing organo-silicates (AO-OM-MMt) were synthesised by a cation-exchange reaction between Na-MMt and an excess of the newly synthesised alkylammonium salt (twice the exchange capacity of the host clay). The quaternary ammonium salt containing the hindered phenol antioxidant (AO-OM) was dissolved in a mixture of ethanol and deionised water 3:1. The solution was sonicated briefly and then left under stirring at $90{ }^{\circ} \mathrm{C}$ (using silicon oil) for $60 \mathrm{~min}$. A $2 \mathrm{wt} \%$ aqueous suspension (deionised water:ethanol = 1:1) of the layered silicate was added to the ammonium salt solution and the mixture was stirred for $5 \mathrm{hr}$ at $90^{\circ} \mathrm{C}$. The cation-exchanged silicates were collected by filtration and subsequently washed with a mixture of hot ethanol and deionised water until a silver nitrate test indicated the absence of chloride anions. The filtered cake was dried at room temperature, ground, and further dried at $70-80^{\circ} \mathrm{C}$ under vacuum for at least $24 \mathrm{~h}$; characterisation of the modified clay will be discussed later (see results). 
An organically modified montmorillonite (OM-MMt) was also prepared (lab-made OM-MMt) by a similar cationexchange reaction between Na-MMt and an excess of the commercially available (not modified) OM, (ETHOQUAD® O/12 PG). The characterization of OM-MMt will be discussed later (see results); comparisons between OM-MMt and the commercial nanofiller, Cloisite ${ }^{\circledR}$ 30B, containing the same OM (ETHOQUAD) are reported at the end as Supplementary Information (see Section S2) at the end of the paper.

\subsection{Processing of the nanocomposites}

All the PA-11 nanocomposites were processed in a Haake MiniLab Micro Compounder model CTW5 fitted with co-rotating twin-screw conical extruder. The polymer melt was continuously processed due to the presence of an integrated bypass valve at the end of the screws that enables the recirculation of the melt via a backflow channel. The pressure in the slit-capillary backflow channel was measured and the torque and pressure versus time curves were continuously recorded. The different clays were added to the molten PA-11 after 2 min of mixing. Processing conditions: Temperature $210^{\circ} \mathrm{C}$, screw speed $100 \mathrm{rpm}$, residence time $5 \mathrm{~min}$. Before processing, the PA-11 and all clays were dried overnight at $90^{\circ} \mathrm{C}$ in a vacuum oven.

\subsection{Testing and Characterisation}

\subsubsection{Fourier Transform Infrared (FT-IR)}

A Fourier Transform Infrared Spectrometer (Spectrum One, Perkin Elmer) was used to record transmission infrared spectra using 16 scans at a resolution of $1 \mathrm{~cm}^{-1}$. Measurements were obtained from an average of triplicate samples with a calculated maximum experimental error (relative standard deviation) of around $5 \%$. FTIR measurements of the different organo-modifiers and the organo-modified clays were carried-out in $\mathrm{KBr}$ disks. FTIR analysis of the PA-11 and its nanocomposites were performed using thin polymer films ( $80 \mu \mathrm{m}$ thick, prepared by compression moulding using a Carver Press at temperature of $210{ }^{\circ} \mathrm{C}$. Before the moulding, the neat PA11 and PA-11-PNCs were dried overnight at $90^{\circ} \mathrm{C}$ in a vacuum oven.). Deconvolution of FTIR peaks was performed using the scientific software OriginPro 2015.

\subsubsection{Gas Chromatography - Mass Spectrometry (GC-MS)}

Agilent 6890 Gas Chromatograph (GC) system equipped with a 30m x 0.250mm HP-5MS GC column and an Agilent 5973 Mass Selective Detector (MSD) detector were used to record the GC-MS spectra of the r-AO (Irganox acid). A Bruker Esquire 3000 PLUS (Ion Trap LC/MS ${ }_{n}$ System) mass spectrometer equipped with electrospray ionization (ESI) source and quadrupole ion trap (QIT) detector was used to record the mass spectrum of AO-OM.

\subsubsection{Proton Magnetic Resonance (1H NMR)}

${ }^{1} \mathrm{H}$ NMR experiments were performed on a Bruker Avance-300 spectrometer at ambient temperature using tetramethylsilane (TMS) as internal standard. The NMR samples were prepared by dissolving $20 \mathrm{mg}$ material 
in $0.7 \mathrm{ml}$ deuterated chloroform $\left(\mathrm{CDCl}_{3}\right)$ solvent which contained $0.05 \% \mathrm{TMS}$. The spectra were recorded at $300.13 \mathrm{MHz}$ using a high-resolution dual $\left({ }^{1} \mathrm{H}{ }^{13} \mathrm{C}\right)$ gradient probe and the $\mathrm{zg} 30$ pulse program with 32 scans and referenced to an internal TMS standard at $0.0 \mathrm{ppm}$.

\subsubsection{Differential Scanning Calorimetry (DSC)}

A Perkin-Elmer DSC7 calorimeter was used. All experiments were performed under dry $\mathrm{N}_{2}$ gas using samples of around $10 \mathrm{mg}$ in $40 \mu \mathrm{l}$ sealed aluminum pans. Four scans (two heating: $30-220^{\circ} \mathrm{C}$ and two cooling: $220-30$ ${ }^{\circ} \mathrm{C}$ ) were performed on each sample at scanning heating/cooling rate of $5^{\circ} \mathrm{C} / \mathrm{min}$.

\subsubsection{Transmission- and Scanning- Electron Microscopy (TEM and SEM)}

The structure of PNC was evaluated qualitatively by characterising the degree of dispersion of the clays in selected nanocomposite samples using TEM. TEM measurements of the extruded samples were recorded on a ZEISS EM 900 transmission electron microscope (with acceleration voltages of 50 and $80 \mathrm{KeV}$ ); all other samples were run on Philips CM120 900 transmission electron microscope. The measurements were done on ultrathin $(70 \mathrm{~nm})$ sections which were obtained at low temperatures $\left(-110\right.$ to $\left.-120^{\circ} \mathrm{C}\right)$ using a LEICA ultra microtome equipped with a diamond knife. The SEM analysis was performed on cryogenically fractured and gold sputtered surfaces of thin compression moulded samples using Philips ESEM XL30 scanning electron microscope.

\subsubsection{Wide Angle X-ray Diffraction (WAXD)}

WAXD was performed on pristine clay and on films to examine the PNC structure (intercalated or exfoliated), the position, shape and intensity of the basal reflections of the silicate layers. Diffraction spectra were obtained over a $2 \theta$ range of $2-15^{\circ}$ using a Siemens D-500 Krystalloflex 810 diffractometer (reflection mode, with Cu-Ka radiation at $\lambda=0.1542 \mathrm{~nm}$ and a scan rate of $1.0 \mathrm{deg} / \mathrm{min}$ ). Quantification of the changes in the interlayer distances on going from the pristine clays to clay-containing PA-11 nanocomposites was carried out according to Bragg's formula ( $d_{001}=n \lambda /(2 \sin \theta)$ where $n$ is an integer and $\theta$ is the angle of incidence of the $\mathrm{x}$-ray beam.

\subsubsection{Rheology}

Rheological tests were performed using a stress-controlled rheometer (Rheometric Scientific, model SR5) in parallel plate geometry (plate diameter $25 \mathrm{~mm}$ ). The complex viscosity $\left(\eta^{*}\right)$, storage $\left(G^{\prime}\right)$ and loss $\left(G^{\prime \prime}\right)$ moduli were measured over frequency scans from $\omega=10^{-1}$ to $10^{2} \mathrm{rad} / \mathrm{s}$ at $\mathrm{T}=210^{\circ} \mathrm{C}$. The strain amplitude was $\mathrm{V}=$ $5 \%$, which preliminary strain sweep experiments proved to be low enough to be in the linear viscoelastic region. Before the analysis, the PA-11-PNCs were dried overnight at $90{ }^{\circ} \mathrm{C}$ in a vacuum oven 


\subsubsection{Thermogravimetry (TG)}

Thermogravimetry was performed on a Netzsch STA 449 F1 Jupiter Simultaneous Thermal Analyser. $40 \mathrm{mg}$ samples were placed in alumina crucibles under nitrogen flow of $20 \mathrm{~m} / / \mathrm{min}$ and heating rate of $10^{\circ} \mathrm{C} / \mathrm{min}$ and scanned in the temperature range of 30 to $600^{\circ} \mathrm{C}$.

\subsection{Thermo-oxidation Stability}

Long Term Thermal Stability (LTTS) tests were carried out in an air-circulating oven at $210^{\circ} \mathrm{C}$ for up to $8 \mathrm{~h}$ for the different clays (incorporated into $\mathrm{KBr}$ disks); PA-11 PNCs were tested at $170^{\circ} \mathrm{C}$ for up to $150 \mathrm{~h}$.

\section{Results and Discussions}

\subsection{Characterization of $A O-O M$ and $A O-O M / M M t$}

A comparison of the FTIR spectra (and the corresponding deconvolution) of the new AO-containing organomodifier (AO-OM) with that of the organomodifier (OM) in the spectral ranges $\left(3700-3100 \mathrm{~cm}^{-1}\right.$ and $1800-1600 \mathrm{~cm}^{-1}$ ) is shown in Fig. 1. The OM spectrum shows one broad absorption peak at about $3325 \mathrm{~cm}^{-1}$ due to intermolecular hydrogen bonding between the two hydroxyl groups in the molecule (O-H Stretch). In the case of AO-OM, a more complex set of peaks appear in this region which, when deconvoluted, reveal two peaks and a shoulder due to the presence of free phenolic O-H and free alcohol O-H groups (at 3646, 3548 $\mathrm{cm}^{-1}$ ) as well as hydrogen-bonded phenolic $\mathrm{O}-\mathrm{H}$ groups (at $3278 \mathrm{~cm}^{-1}$ ). Similarly, examining the carbonyl and unsaturation region in the range 1800-1400 $\mathrm{cm}^{-1}$, shows that in the case of the OM there is one broad absorption peak at $1640 \mathrm{~cm}^{-1}$ due to the unsaturation in the long alkyl tallow chain, whereas the AO-OM spectrum shows not only the peak at $1640 \mathrm{~cm}^{-1}$ of the tallow, but also the appearance of a new peak at 1740 $\mathrm{cm}^{-1}$ attributed to the ester groups of the reacted hindered phenol antioxidant molecules within the AO-OM structure.

Comparison of the ${ }^{1} \mathrm{H}$ NMR of the AO-OM with that of OM (Fig. 2) shows clearly that in the former case, in addition to the presence of characteristic signals of the parent $\mathrm{OM}$, e.g. methyl group on the quaternary nitrogen (proton $\mathbf{f}$ ) and the terminal methyl group in the tallow chain (proton $\mathbf{w}$ ), a number of new signals appear. To characterise these, the ${ }^{1} \mathrm{H}-\mathrm{NMR}$ spectrum of AO-OM (Fig. 2 ) is compared with that of Irganox acid (see Supplementary Information, Fig. S1A). It is clear that the new signals correspond to chemical shifts of the hindered phenol antioxidant moiety present in the AO-OM molecule as illustrated by the presence of methyl groups of the tertiary butyl substituents on the hindered phenol rings (protons $\mathrm{E}^{\prime}$ ), the methylene groups of the propionate group of the hindered phenol (protons $\mathrm{C}^{\prime}$ and $\mathbf{D}^{\prime}$ ) and the $\mathrm{C}-\mathrm{H}$ groups in the benzene ring (protons $A^{\prime}$ ). This confirms clearly that the esterification reaction between the $-\mathrm{COOH}$ in the $\mathrm{r}-\mathrm{AO}$ with the $-\mathrm{OH}$ in the $\mathrm{OM}$ has taken place giving rise to a chemically attached $\mathrm{AO}$-containing organic modifier, $\mathrm{AO}-\mathrm{OM}$. 


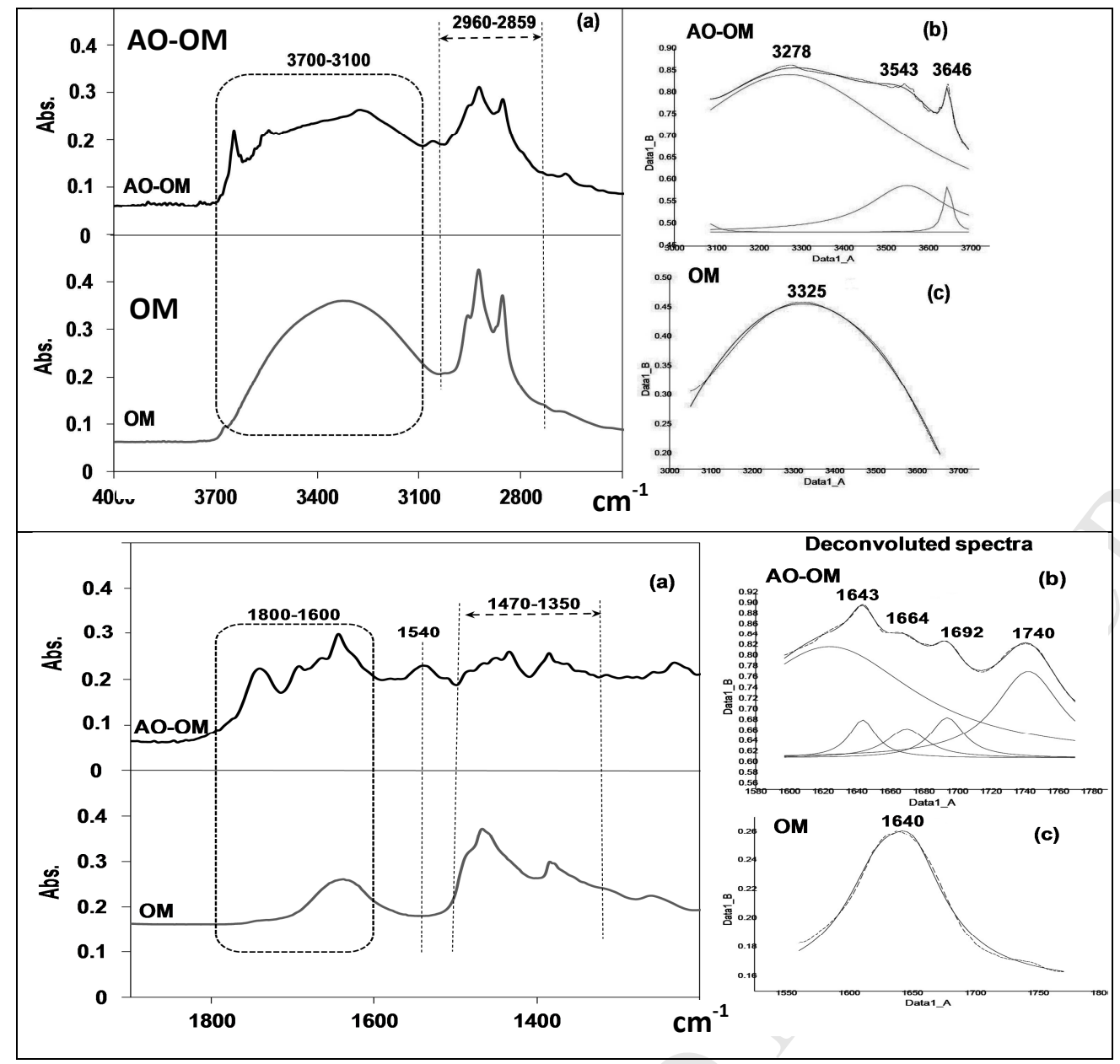

Fig. 1

NMR and MS analyses have also confirmed that the synthesised AO-OM product is a mixture comprising one and two hindered phenol antioxidant molecules (see structures in Fig. 2). Evidence from ${ }^{1} \mathrm{H}$ NMR for the presence of a one (AO-OM1) and two (AO-OM2) hindered phenol antioxidant groups in the AO-OM product mixture can be gleaned from a comparison of the integration values of a number of signals (integration is directly proportional to the number of protons ascribed to the signal). The NMR integration results suggest that, in the AO-OM product mixture, for every two tallow chains there is at least three hindered phenol moieties suggesting that the mixture contains both AO-OM1 (containing one hindered phenol) and AO-OM2 (having two hindered phenols), see Fig. 2 for structures. This conclusion is based on comparison of the integration of six protons (of the terminal $\mathrm{CH}_{3}$ groups (protons $\mathbf{w}, \delta=0.9 \mathrm{ppm}$ ) in two tallow chains of the AO-OM mixture (integration value $=6.00$ ) with that of the propionate methylene protons $\mathrm{D}^{\prime}, \delta=2.7 \mathrm{ppm}$ (in the hindered phenol moiety) which have an integration value slightly higher than six (at 6.88), and similarly in comparison with the integration value of the other propionate methylene protons of the hindered phenol, (protons $\mathbf{C}^{\prime}, \delta=2.9 \mathrm{ppm}$, with a value of 6.48$)$. This supports the suggestion that there are at least three methylene groups $\left(\mathrm{CH}_{2}\right.$ with protons C' or protons D') originating from three hindered phenol moieties, see Fig.2, indicating the presence of both AO-OM1 and AO-OM2 in the AO-OM mixture. 
Further confirmation for the presence of a mixture of molecules (AO-OM1 and AO-OM2) is obtained from the integration of the methyl groups in the tertiary butyl substituents on the hindered phenol rings (protons E'; $\delta=1.45 \mathrm{ppm}$ ) which give 54 protons corresponding to $18 \mathrm{CH}_{3}$ groups in the t-Bu substituents of the AO-OM mixture (originating from 3 hindered phenols). Integration of signals of the methylene group located next to the hydroxyl oxygen of the quaternary ammonium salt (protons $b$ in OM and also in AO-OM1) and the methylene protons next to the ester oxygen (protons $\mathbf{b}_{1}{ }_{1}$ in AO-OM1 and $\mathbf{b}_{2}{ }_{2}$ in AO-OM2) sheds further light on the overall structure of the AO-OM product mixture. Fig. 2 shows that for the $\mathrm{OM}$, there is only one $\mathrm{CH}_{2}$ signal corresponding to protons $\mathbf{b}$ with $d=4.03\left(\mathrm{CH}_{2}\right.$ next to the $\mathrm{OH}$ groups), whereas in the AO-OM mixture, in addition to the corresponding methylene group signal (protons $\mathbf{b}$ in AO-OM1 at $4.07 \mathrm{ppm}$ ), there are two clearly separated additional signals, one corresponding to the methylene group in AO-OM1 ( $\mathbf{b}_{1}{ }_{1}$ at $\delta=4.58$ $\mathrm{ppm}$ ) and the other corresponding to the two methylene groups in AO-OM2 (b'2 at $\delta=4.15 \mathrm{ppm}$ ). Further examination of the integrated signals of the propionate methylene groups in the hindered phenol moiety (protons $\mathbf{b}^{\prime}$ ) of the AO-OM mixture gives a good indication of the relative ratio of AO-OM1 to AO-OM2 in the AO-OM product mixture. Fig. 2 shows two signals for protons b' ( $\mathbf{b}^{\prime}{ }_{1}$ and $\mathbf{b}{ }_{2}{ }_{2}$ ) with $\mathbf{b}_{2}{ }_{2}$ appearing at chemical shift of 4.15 ppm and having an integral of 4.97 due to 4 protons (for AO-OM2), and protons b' ${ }_{1}$ appearing at $\delta=4.58 \mathrm{ppm}$ with an integral of 2.17 due to 2 protons (for AO-OM1). Based on these NMR results, the ratio of $\mathrm{AO}-\mathrm{OM} 1$ to AO-OM2 in the mixture is about $1: 1.13(47: 53)$.

Qualitatively, MS results lends further support for the composition of the AO-OM product mixture; three groups of signals indicating the presence of three distinctly different molecular species. The presence of the first and the second groups of signals (labelled $\mathbf{A}$ and $\mathbf{B}$ in Fig. 3 ) is attributed to the AO-OM mixture of molecules having two antioxidant functionalities (group A showing larger amount of AO-OM2 in the mixture; Mw=891.4 $\mathrm{g} / \mathrm{mol}$, formula: $\mathrm{C}_{57} \mathrm{H}_{96} \mathrm{NO}_{6}$ ) and one antioxidant functionality (group B corresponding to AO-OM1; $\mathrm{Mw}=631.0$ $\mathrm{g} / \mathrm{mol}$, formula: $\mathrm{C}_{40} \mathrm{H}_{72} \mathrm{NO}_{4}$ ). The third group of signals (group $\mathrm{C}$ ) indicates the presence of small fragments including those from the unreacted $\mathrm{OM}\left(\mathrm{M}_{\mathrm{W}}=370\right)$. It is interesting to point out here that in spite of the use of a large excess of the Irganox acid during the synthesis of AO-OM (AO:OM molar ratio of 3:1), it does not seem to have prevented the formation of AO-OM containing only one antioxidant molecule (AOM1) but the overall results do suggest that there is a slight excess of AO-OM2 in the mixture. 


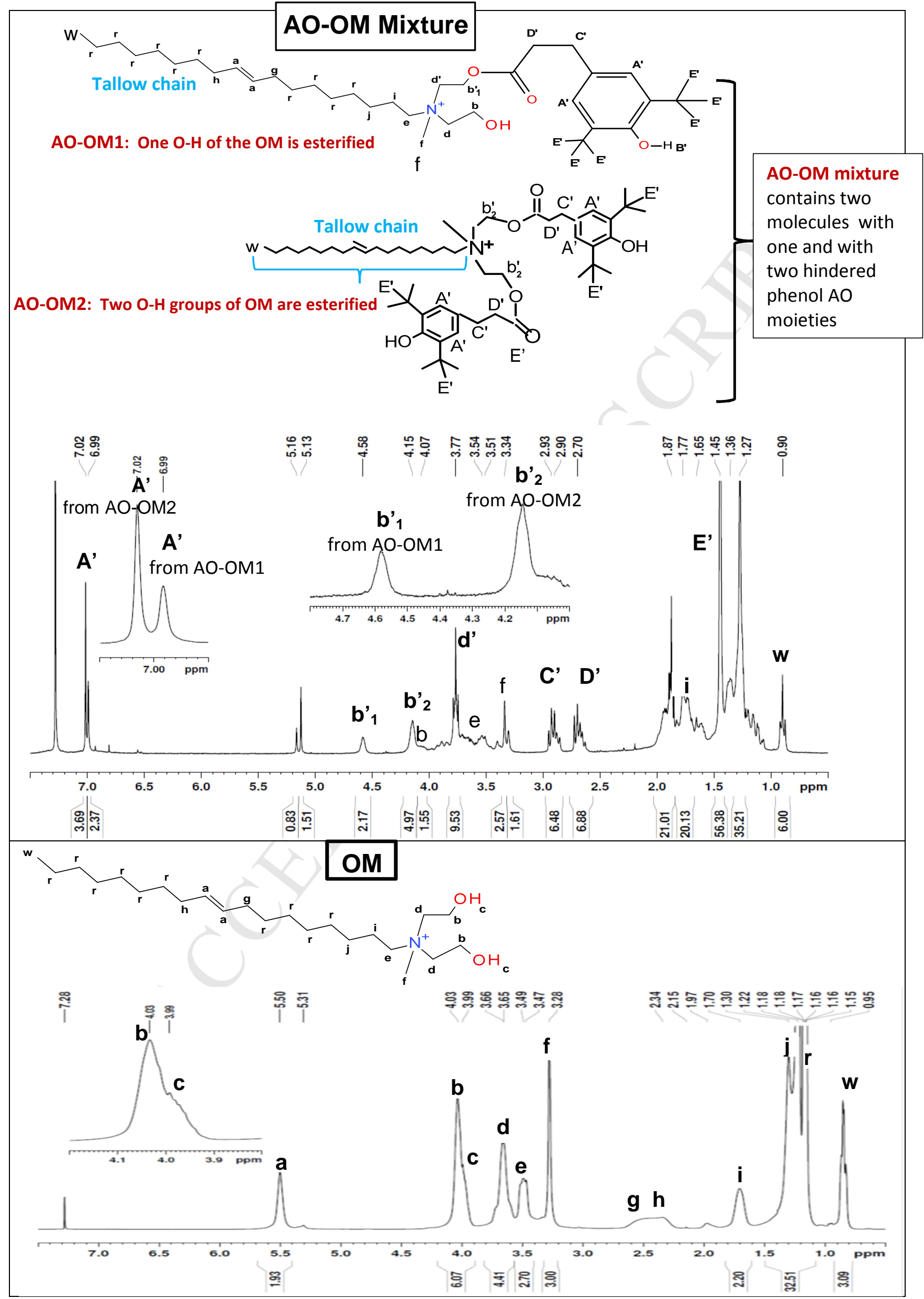

Figure 2 


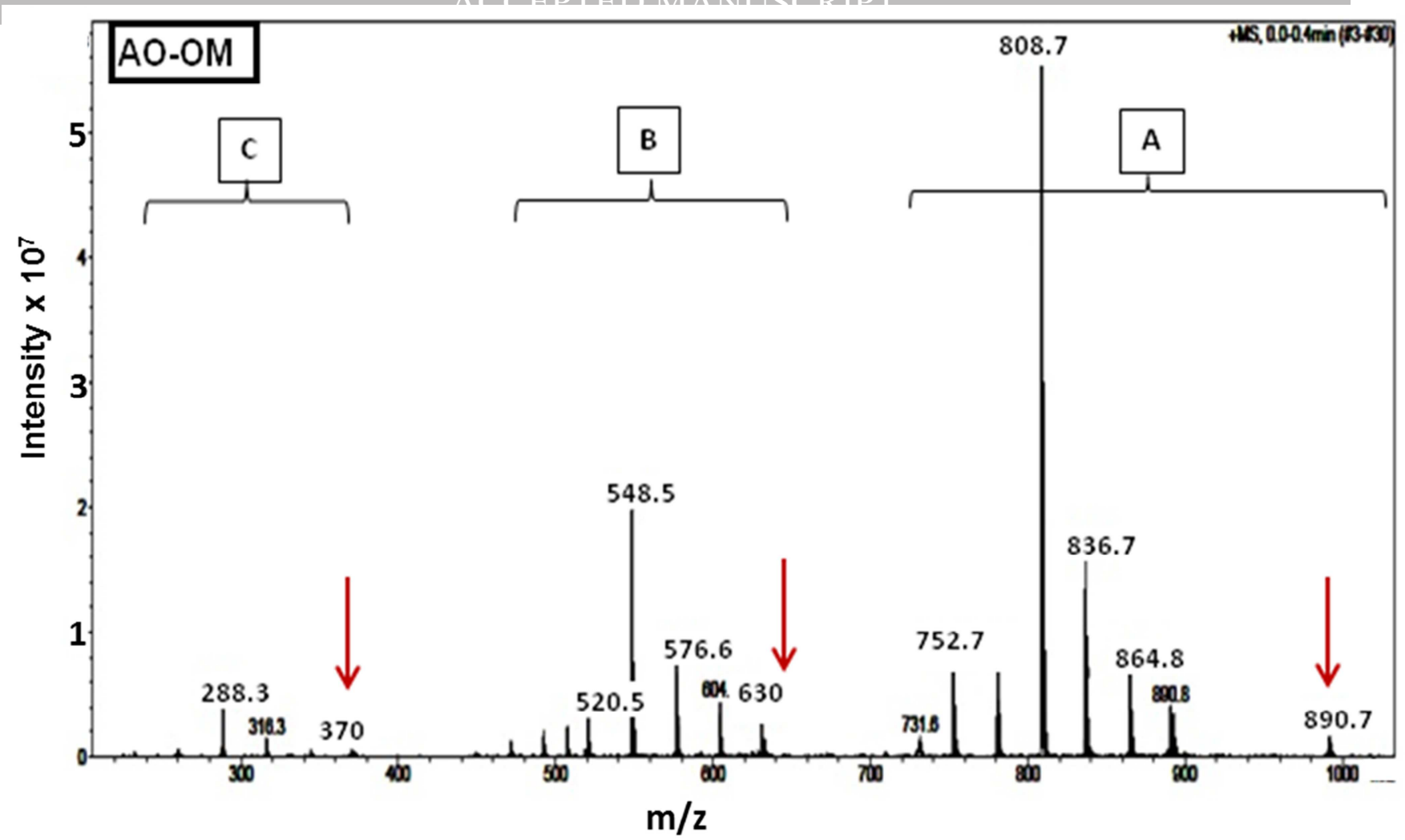

Figure 3

In order to prepare the AO-containing organo-silicates (AO-OM-MMt), the AO-OM was intercalated in Na-MMt by cation exchange reaction. Fig. 4a shows the FTIR spectra in the range $1900-1200 \mathrm{~cm}^{-1}$ of the newly prepared AO-OM-MMt, as well as that of Na-MMt and the laboratory-prepared OM-MMt clays; the corresponding deconvoluted peaks are given in Figs. 4b and 4c. The $1640 \mathrm{~cm}^{-1}$ absorption peak which is observed in all the clays is more prominent in the Na-MMt but appears only as a small shoulder in the two OMcontaining MMt clays. This peak is attributed to the bending mode of vibration of water molecules in the clay structure; the smaller absorption peaks observed in the organo-modified clays is almost certainly due to the lower amount of water molecules present in the coordination of the organo-modifier cations that replace the $\mathrm{Na}^{+}$cations after the cation-exchange reaction. Fig. 4 shows also absorption peaks in the range of 1490 to $1350 \mathrm{~cm}^{-1}$ which can be seen in all the organically-modified clay spectra and is in agreement with the literature [32]. These peaks are attributed mainly to the vibrational stretching of -C-C- (1488 and $\left.1460 \mathrm{~cm}^{-1}\right),-\mathrm{C}-\mathrm{H}(1470$ $\left.\mathrm{cm}^{-1}\right),-\mathrm{CH}_{3}\left(1437 \mathrm{~cm}^{-1}\right)$ groups, and to the in-plane bending of $-\mathrm{CH}_{2^{-}}\left(1455-1362 \mathrm{~cm}^{-1}\right)$ group [32]. However, in the case of AO-OM-MMt, a new additional absorption peak appeared was observed to occur at $1738 \mathrm{~cm}^{-1}$ (which corresponds to the $\mathrm{C}=\mathrm{O}$ stretching of the ester group in the hindered phenol structure) and confirms further a successful intercalation of AO-OM into the clay.

The FTIR spectra of the clays in the $3700-2700 \mathrm{~cm}^{-1}$ region are shown in Fig. 4. Deconvolution of the spectra in the $\mathrm{CH}$ stretching vibration range reveals three different well resolved and separated absorption peaks 
corresponding to $-\mathrm{CH}_{2}$ and $-\mathrm{CH}_{3}$ groups at around 2855, 2931 and $2965 \mathrm{~cm}^{-1}$. The peak at $2965 \mathrm{~cm}^{-1}$ (assigned to the $-\mathrm{CH}_{3}$ stretching vibration) appears distinctly in the case of AO-OM-MMt sample (see Fig. 5 b), whereas it appears only as a shoulder for the lab-made OM-MMt clay sample (see Fig. 5 c). This confirms the presence of a larger amount of methyl groups in the AO-OM-MMt sample compared to the OM-MMt clay which is almost certainly due to the presence of additional methyl groups due to the t-Bu groups of the hindered phenol AO within the structure of the new OM. Moreover, the successful cation-exchange reaction for the preparation of OM-MMt was also confirmed from the similarity in the FTIR spectra of the lab-prepared OMMMt to that of the commercial Cloisite $₫ 30 \mathrm{~B}$ clays (both contain the same OM), see Supplementary Information Section S2.

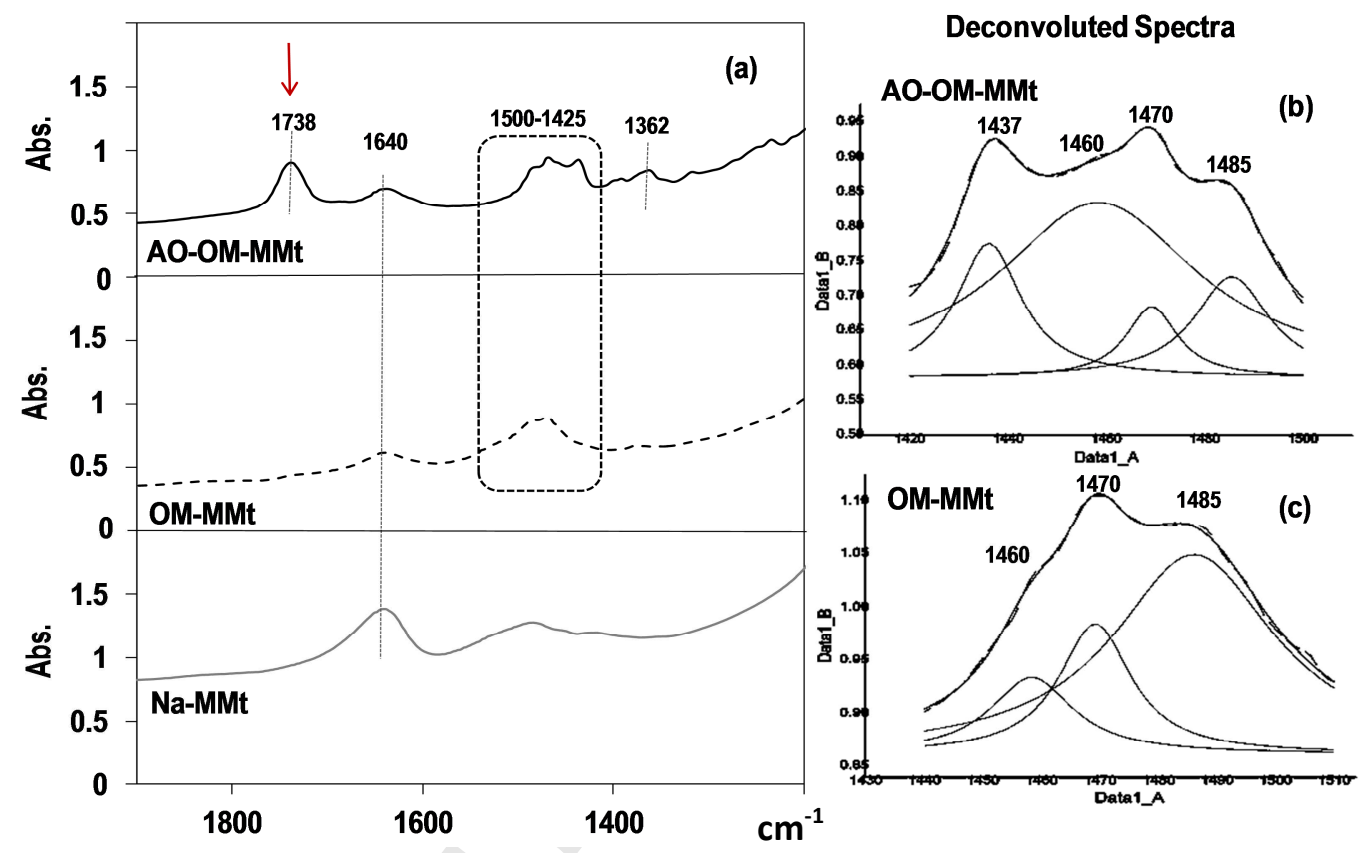

Figure 4

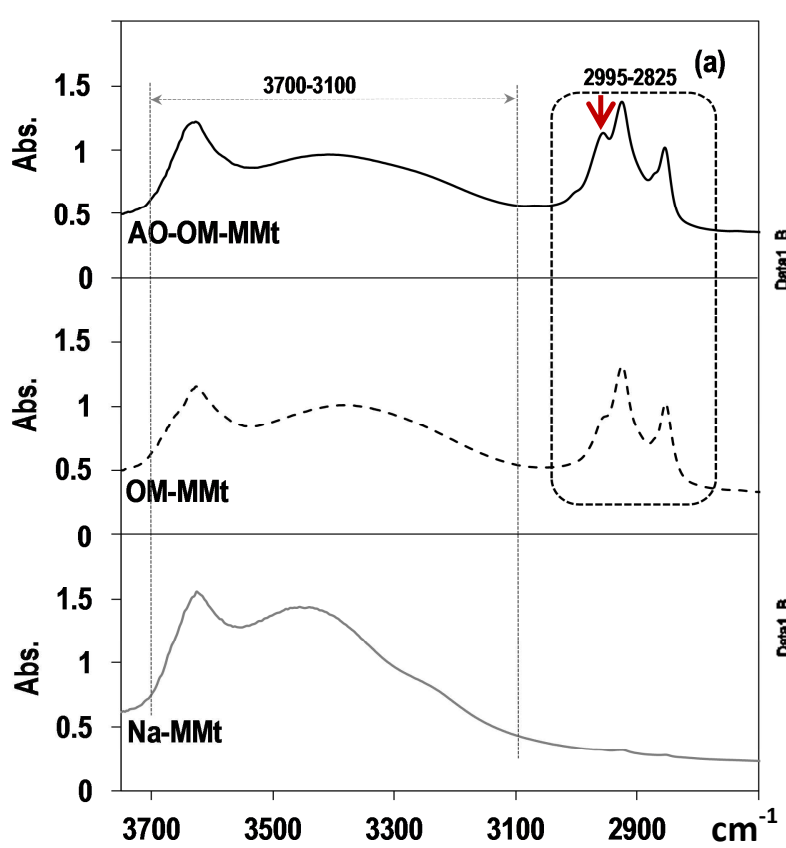

Deconvoluted Spectra
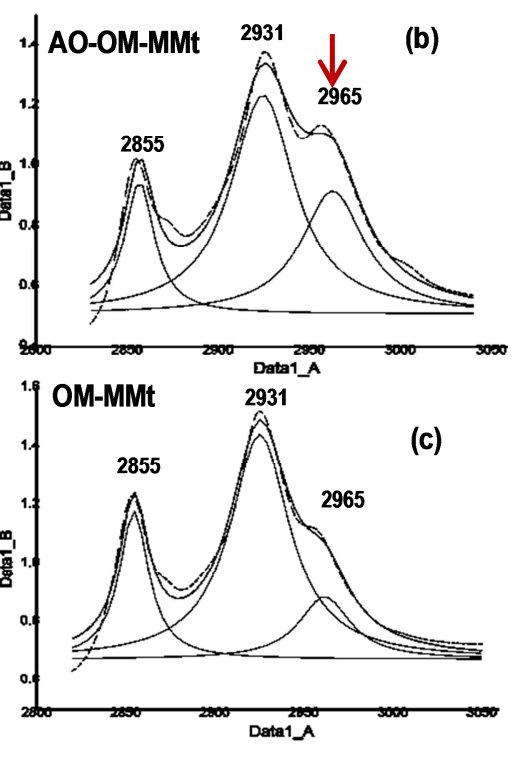

Figure 5 
XRD characterization of the AO-OM-MMt was also performed and the diffraction patterns are shown in Fig. 6. The calculated interlayer distance for AO-OM-MMt is d d001 $=2.05 \mathrm{~nm}$ (main peak $2 \theta=4.30 \mathrm{deg}$ ), while that for the OM-MMt is $1.85 \mathrm{~nm}$ (main peak $2 \theta=4.76 \mathrm{deg}$; similar to the manufacture's data, see Table 1 ) and NaMMt is $1.18 \mathrm{~nm}$ (main peak $2 \theta=7.50 \mathrm{deg}$ ); similar to the manufacture's data). The larger interlayer distance for AO-OM-MMt compared to that of the OM-MMt sample can be explained in terms of the greater steric hindrance engendered by the $\mathrm{AO}-\mathrm{OM}$ molecule compared to the much smaller $\mathrm{OM}$ group.

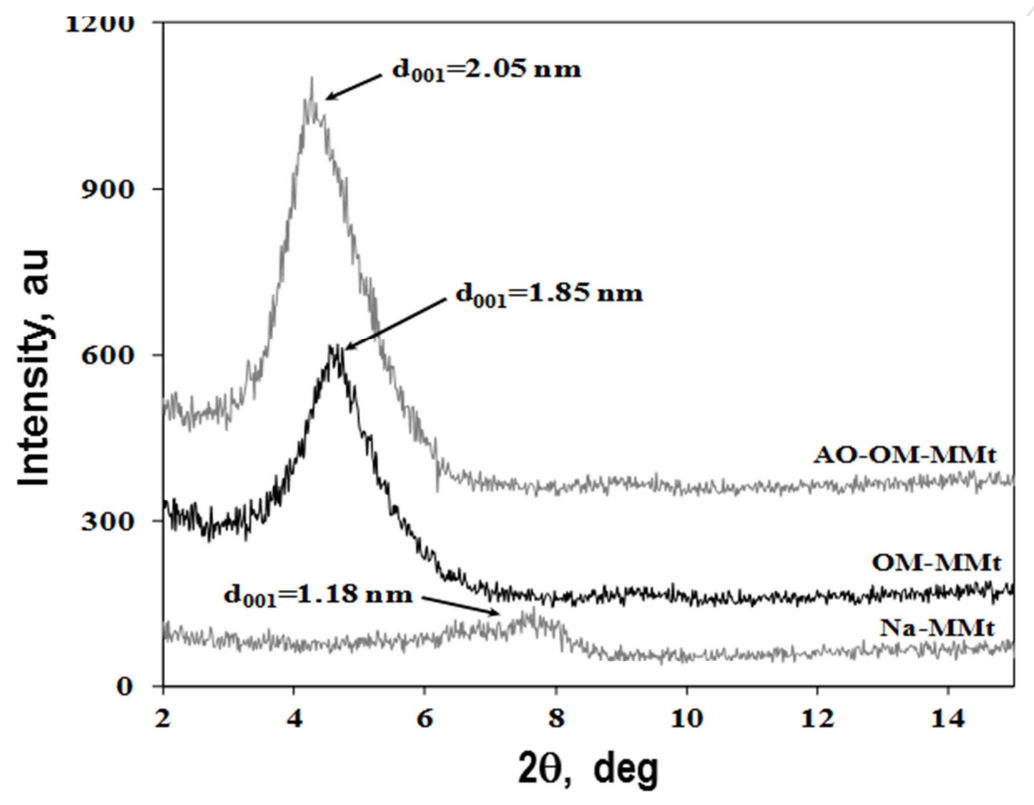

Figure 6

To investigate the amount of $\mathrm{OM}$ in AO-OM-MMt, thermogravimetric (TG) experiments were performed. Figure 7 shows the weight loss curves as a function of temperature for the AO-OM-MMt sample along with that of OM-MMt and Na-MMt. Furthermore, the lab-prepared OM-MMt clay gave similar weight loss curve to that of the commercial Cloisite $\circledast 30 B$ clay (see Supplementary Information Section, Fig. S2b). The Na-MMt shows a gradual weight loss of about $10 \%$ at temperature over $100{ }^{\circ} \mathrm{C}$ (due to the volatilization of water molecules coordinated by the silicate layers). The volatilization of water molecules in both the AO-OM-MMt and OM-OMMt samples is less pronounced due to the presence of the organo-modifier. Moreover, the TG curves of $\mathrm{AO}-\mathrm{OM}-\mathrm{MM}$ and $\mathrm{OM}-\mathrm{OMM}$ samples show a gradual weight loss related to the thermal decomposition of the organic component in the range $280-400^{\circ} \mathrm{C}$, i.e. well above the temperature at which the nanocomposites were melt processed. The residues at the end of the analysis were $74.9 \mathrm{wt} \%$ and $65.8 \mathrm{wt} \%$ for OM-MMt and AO-OM-MMt, respectively, whereas a residual amount of $89.1 \mathrm{wt} \%$ was found for the Na-MMt due to a water loss and partial decomposition of the silicate material, see Fig. 7. The organic content in OM- 
MMt and AO-OMMt is, therefore, about $14.2 \mathrm{wt} \%$ and $23.3 \mathrm{wt} \%$, respectively. Based on this, the overall content of $\mathrm{AO}$ in the AO-OM-MMt clay (based on the gradual mass loss of the AO-OM-MMt) is estimated to be around 9.1 wt \% (see Fig. 7).

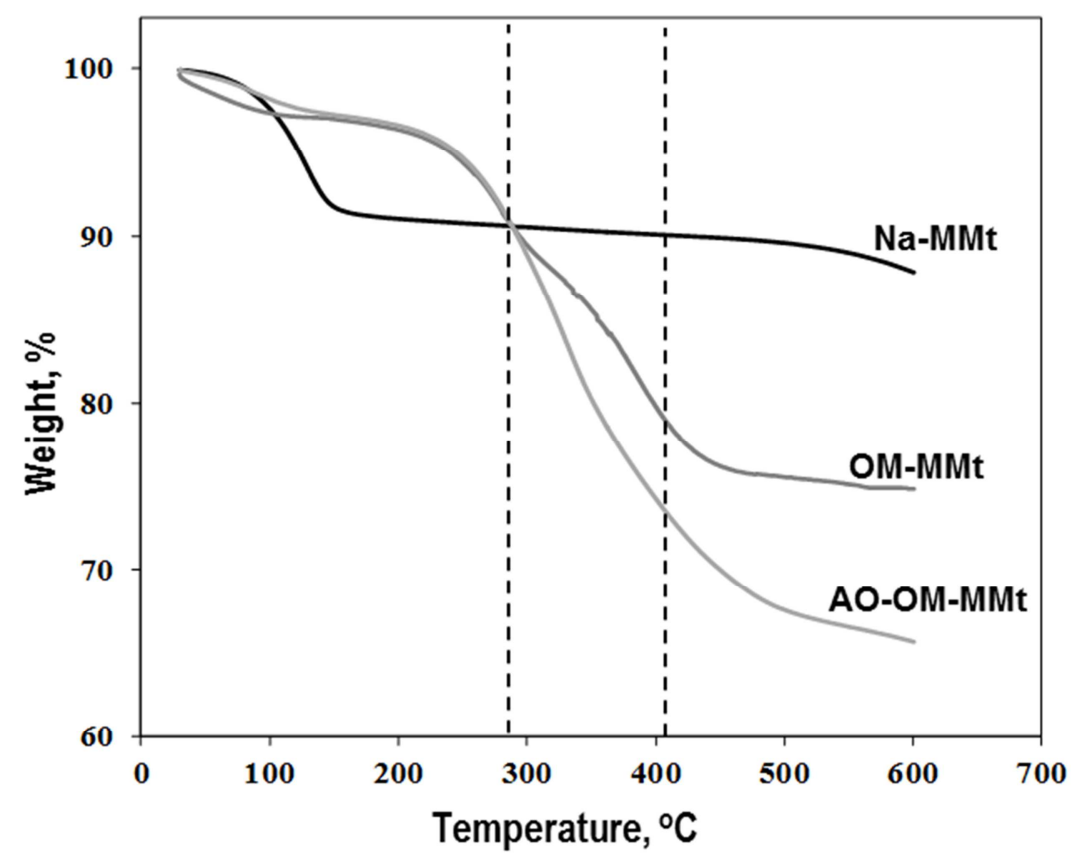

Figure 7

\subsection{Thermo-oxidative Stability of AO-OM-MMt}

The thermo-oxidative stability of the new AO-OM-MMt clay was assessed by accelerated thermal ageing test (at $210^{\circ} \mathrm{C}$ for $480 \mathrm{~min}$ ) and the results are compared with those of the unmodified Na-MMt and the lab-made OM-MMt, i.e. without the antioxidant. Figure 8 shows the FTIR spectra at different ageing times of the different clays; on ageing (up to $480 \mathrm{~min}$ ), all the clays show a gradual loss of water present in the interlayer spaces, see range (1) in the spectra (a-c). For the Na-MMt, the peak at about $1640 \mathrm{~cm}^{-1}$ (see range (3) in spectra (a)) shifts to lower wavenumber with ageing but remains a distinct albeit less intense after $480 \mathrm{~min}$. The organo-modified clay samples, on the other hand, show only a shoulder at $1640 \mathrm{~cm}^{-1}$ before ageing, and is almost unaffected by the thermal treatment. 

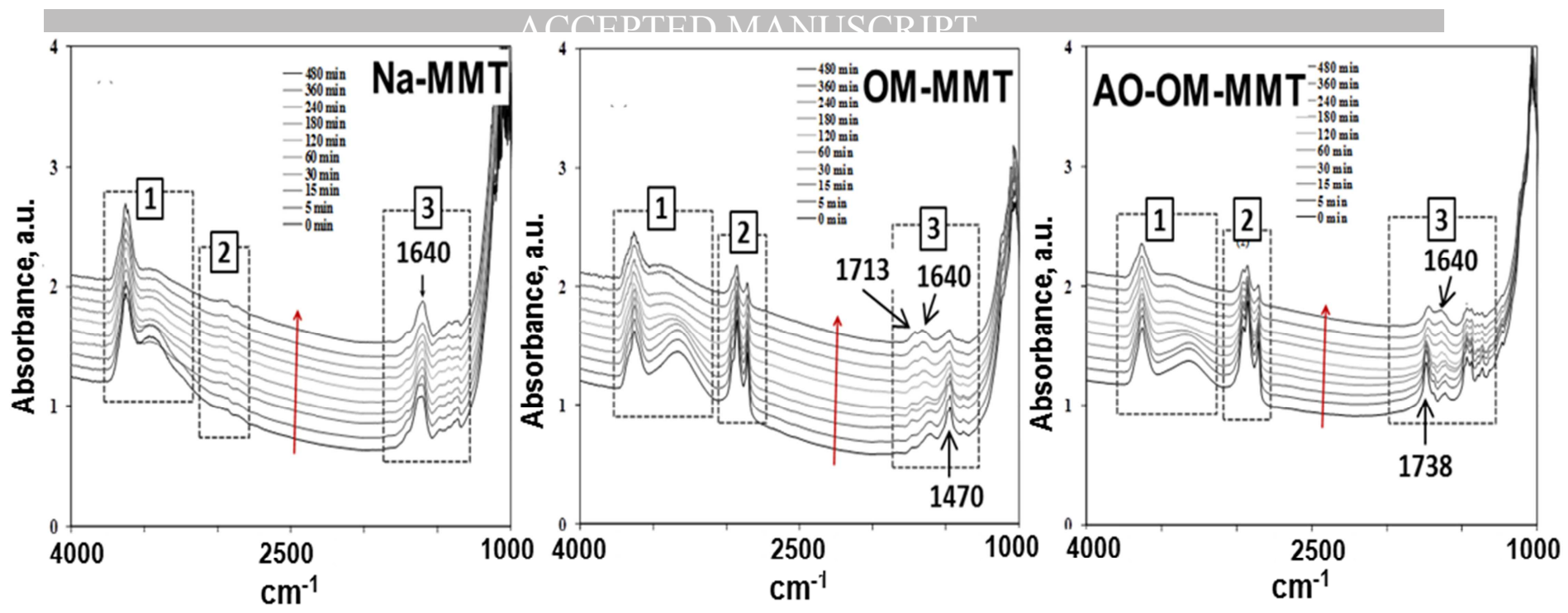

Figure 8

The decrease of the infrared peaks (attributed to the $-\mathrm{CH}_{2}$ and $-\mathrm{CH}_{3}$ stretching vibrations (around $1470 \mathrm{~cm}^{-1}$, see range (3) and around 2855, 2931 and $2965 \mathrm{~cm}^{-1}$, see range (2) in Figs. 8b and 8c) with ageing is similar for all the clays containing OM which suggests similar patterns in their thermo-degradation profile. It is well known that the thermal degradation of the ammonium-based OM follows the Hoffman elimination mechanism which leads to the formation of a-olefins, ammonium salts and low molecular weight species [24]. In the labmade OM-MMt, a peak at around $1713 \mathrm{~cm}^{-1}$ is formed (absent in NaMMt); its origin is, most likely, the acidic degradation species formed from OM via the Hoffman process. The spectra of the AO-OM-MMt, Fig. 8c, range (3), shows initially the antioxidant ester absorption peak at $1738 \mathrm{~cm}^{-1}$ which decreases with ageing time. It is worth mentioning here, however, that during thermal degradation, this AO-OM-MMt can be expected to release by-products with active antioxidant functionalities within the confines of the clay galleries thereby creating a 'reservoir' of stabilising species which can be released subsequently to offer the additional stabilisation found in these nanocomposites.

\subsection{Processing, Morphological and Calorimetric Characterizations of PA-11-Nanocomposites}

PA-11 nanocomposites containing different clays, the Na-MMt and the different organo-modified clays, in the absence and presence of a reacted or an added (i.e. free not chemically reacted) hindered phenol antioxidant, were prepared by melt mixing. Figure 9 shows the torque generated during the melt extrusion of the different PA-11 nanocomposites reflecting changes in their melt viscosity. It is clear that all the nanocomposites prepared without antioxidants show higher torque values than the neat PA-11 (without antioxidant), whereas, both stabilised nanocomposites samples, PA-11/AO-OM/MMt and PA-11/OM-MMt//rg, show lower overall torque values, with the former (containing reacted $\mathrm{AO}$ ) giving the lowest torque/melt viscosity indicating a better melt stability. Thermoxidative degradation mechanisms of neat PA11 were shown [33] to result in increase in molecular weight due to predominantly crosslinking reactions that take place through 
recombination of broken chains (formed through initial chain scission processes) and/or post condensation reactions. Furthermore, it has also been shown recently [34] that the presence of organo-clay in PA11 matrix give rise to an accelerated rate of the crosslinking reactions. These literature findings fully support the melt processing torque results shown in Fig. 9 whereby the organo-clay modified nanocomposites (without stabilisation) give higher torque values than PA11 indicating higher melt viscosities and molecular weight due to faster rate of the matrix crosslinking. In contrast, the reduction in the torque shown by the stabilised nanocomposites confirm the stabilising effect resulting in an overall lower extent of these crosslinking degradative reactions.

Further, it has been shown [35] that the dispersion morphology of the clay particles has a remarkable influence on the melt rheological properties of PA11 and the nanocomposites. Accordingly, it is suggested here, that the PA-11/AO-OM/MMt sample may show good clay dispersion and intercalated/exfoliated morphology (see TEM and SEM results later).

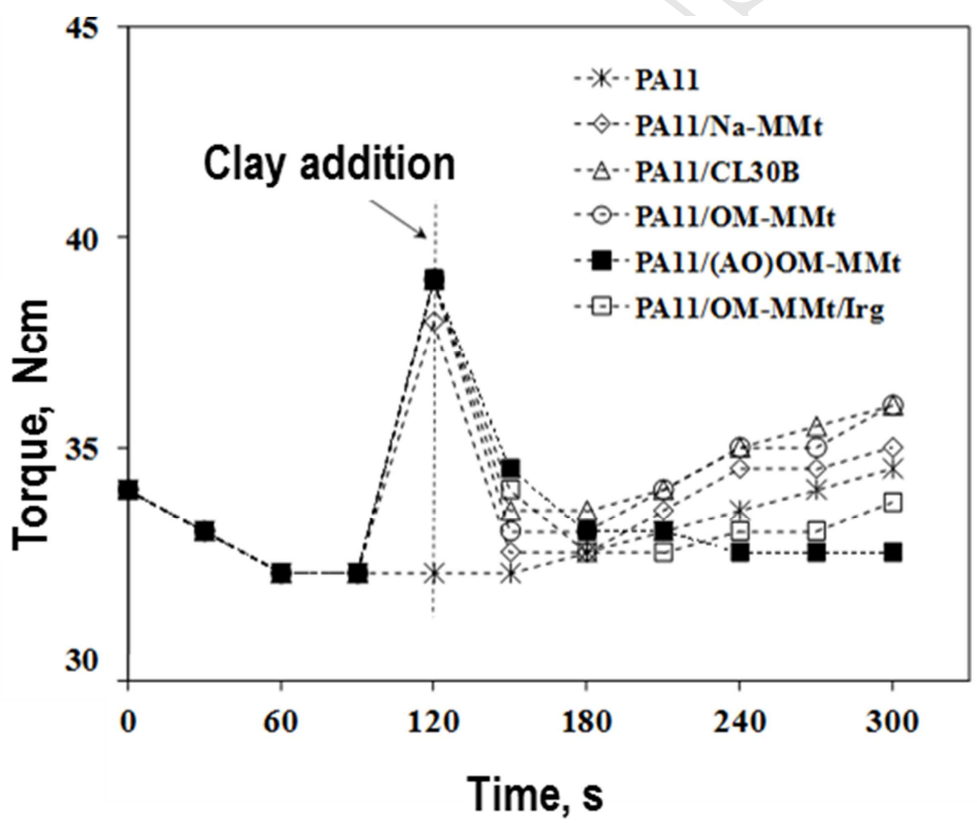

Figure 9

The morphology after processing of the different PA-11-based nanocomposites was investigated by XRD, SEM and TEM. Fig.10 shows the x-ray diffraction patterns of the nanocomposites. It is clear that while the PA-11/Na-MMt displays a visible diffraction peak at about $2 \theta=7.50$ deg due to the presence of Na-MMt stacks, all the PA-11-based OM-MMt nanocomposites did not show such distinguishable diffraction peak, suggesting the formation of predominantly exfoliated and/or intercalated clay morphology. In all the organically modified samples, only small shoulders can be seen in their XRD patterns at about $2 \theta=7.50$ deg and this may be due to the presence of a minimal fraction of unmodified MMt stacks, originating from the pristine clay and/or due to the small collapsed clay structures after the removal of the OM [35]. In the case of the PA-11/AO-OM-MMt, the 
XRD shows the smallest shoulder in this region suggesting the presence of the lowest amount of collapsed MMt stacks. This may be due to the large steric hindrance within the structure of the new AO-OM making the MMt stackes in this case less likely to collapse.

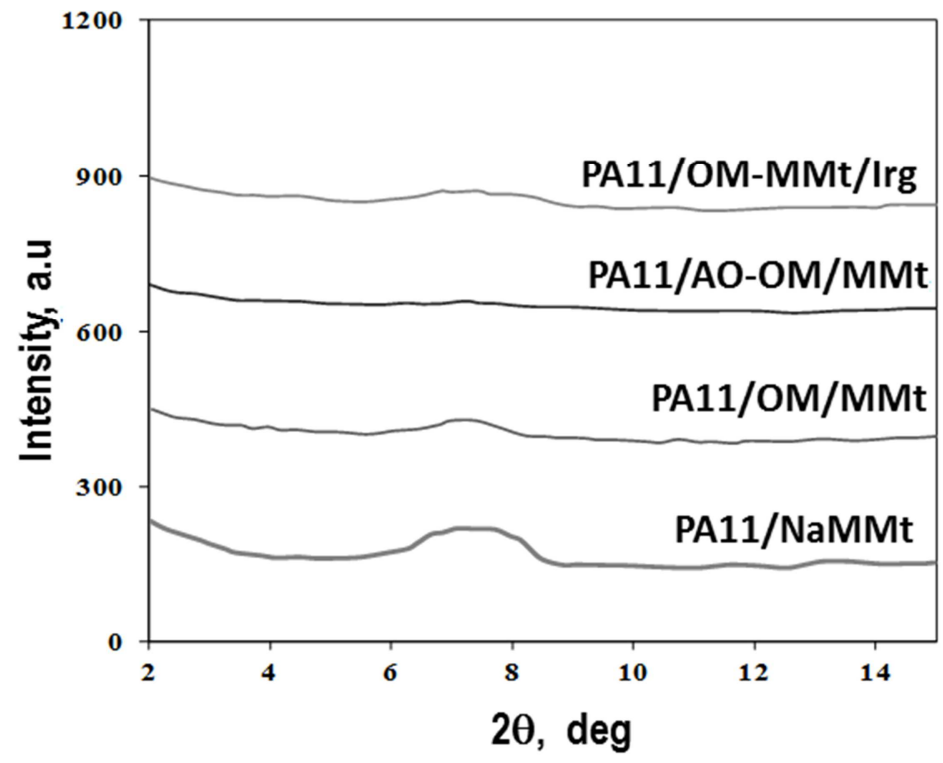

Figure 10

The overall clay nanostructure morphology was examined and Figure 11 shows the TEM of the different nanocomposites samples. The TEM micrographs for both the unstabilised PA-11/OM-MMt (Figs.11 e-f) and the stabilised (with the reacted antioxidant) PA-11/AO-OM-MMt samples (Figs. $11 \mathrm{~h}$-i) show clearly exfoliated clay morphologies, with the clay platelets in the stabilised sample (PA-11/AO-OM-MMt) appearing as smaller and more well dispersed compared to the unstabilised (PA11/OM-MMt) sample; the PA-11/Na-MMt sample shows poorly dispersed clay stacks with different micrometric dimensions (Figs. 11 b-c). Further, SEM was also used to examine the state of dispersion of the clay in the PA11-nanocomposites samples, and the micrographs show also the poor dispersion, as excpected, of the Na-MMt clay, Fig. 11a (large silicate particles of $\sim 2 \mu$ ) compared to the much better state of clay dispersion exhibited by the OM-containing PA-11 nanocomposite samples (see Figs. 11d and 11g). The better clay dispersion and exfoliation in the stabilised samples must be responsible for the disappearance of the XRD characteristic peaks (Fig. 10). The excellent clay dispersion in the PA-11/AO-OM-MMt sample (clearly seen from its TEM, Fig. $11 \mathrm{~h}$ and $\mathbf{i}$ ) could be a consequence of the greater interlayer distances between the platelets that helps with the introduction of the polymeric macromolecules between the silicate layers. This morphology, along with the presence of a reacted hindered phenol antioxidant moieties within the clay structure, could then have a direct beneficial effect on stabilising the melt, especially at the interface region between the inorganic clay phase and the matrix. 


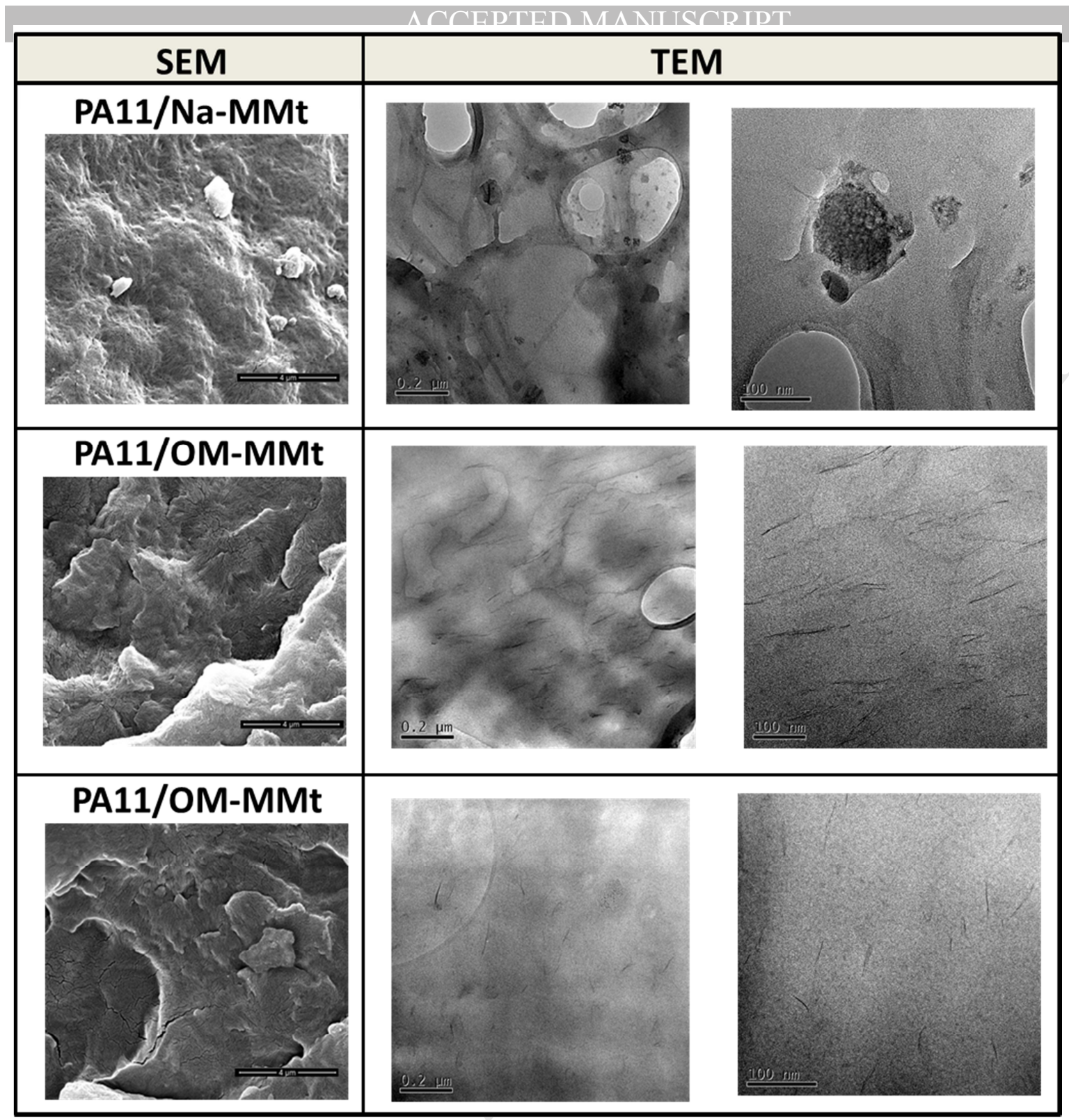

Figure 11

Differential scanning calorimetric analysis (see Fig. 12 \& Table 2) shows that the differently modified clays do not influence the melting temperature but lead to a slight decrease in the melting enthalpy in the first and second heating scans. Data presented in Table 2 indicate that all the clays used do not show any major nucleating effect since the crystallization temperatures and enthalpies (enthalpy values corrected for the clay content based on both first and second heating scans) of all the PA-11-PNCs show only a small change compared to those of the neat PA-11. The neat PA sample (from the second heating scan) shows a main melting peak at about $186.4{ }^{\circ} \mathrm{C}$ (a-form, zigzag chain conformation), and only a just detectable shoulder at about $180^{\circ} \mathrm{C}$ due to the $y$-crystalline (twisted) form [36-38]. It is worth noting that the organically-modified claycontaining PNC samples show more pronounced features at about $180^{\circ} \mathrm{C}$, compared to the neat PA-11. The appearance of these features may be attributed to a greater ease of formation of the $y$-crystal in these samples [38] as it is well known [39] that the formation of $y$-crystals is associated with the PA chain alignment on the clay layers. 


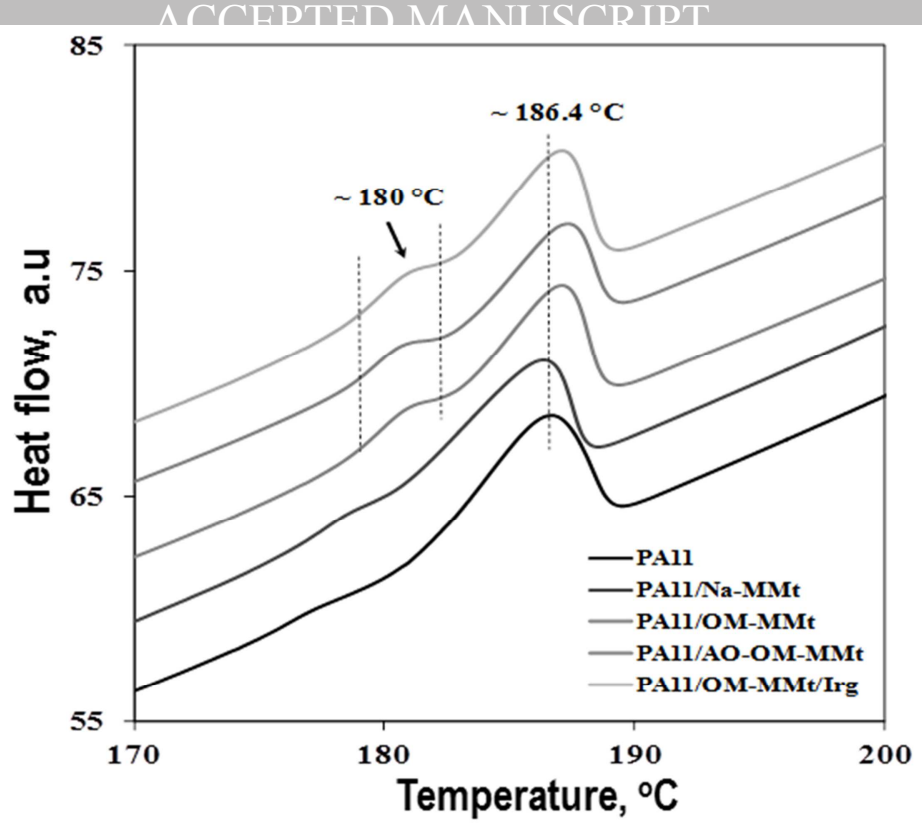

Figure 12

Table 2. Melting $\left(\mathrm{T}_{\mathrm{m}}{ }^{\circ} \mathrm{C}\right)$ and crystallization $\left(\mathrm{T}_{\mathrm{c}}{ }^{\circ} \mathrm{C}\right)$ temperatures and melting $\left(\Delta \mathrm{H}_{\mathrm{m}}\right)$ and crystallisation $\left(\Delta \mathbf{H}_{c}\right)$ enthalpies (corrected for the clay content) for neat PA-11 and PA-11PNCs (from DSC)

\begin{tabular}{|l|c|c|c|c|c|c|c|c|}
\hline \multirow{2}{*}{ Sample ID } & \multicolumn{2}{|c|}{ 1st Heating } & \multicolumn{2}{c|}{ 1st Cooling } & \multicolumn{2}{c|}{ 2nd Heating } & \multicolumn{2}{c|}{ 2nd Cooling } \\
\cline { 2 - 9 } & $\begin{array}{c}T_{m} \\
\left({ }^{\circ} \mathrm{C}\right)\end{array}$ & $\begin{array}{c}\Delta H_{m} \\
(\mathrm{~J} / \mathbf{g})\end{array}$ & $\begin{array}{c}T_{\mathrm{c}} \\
\left({ }^{\circ} \mathrm{C}\right)\end{array}$ & $\begin{array}{c}\Delta H_{\mathrm{c}} \\
(\mathrm{J} / \mathbf{g})\end{array}$ & $\begin{array}{c}T_{m} \\
\left({ }^{\circ} \mathrm{C}\right)\end{array}$ & $\begin{array}{c}\Delta H_{m} \\
(\mathrm{~J} / \mathbf{g})\end{array}$ & $\begin{array}{c}T_{\mathrm{c}} \\
\left({ }^{\circ} \mathrm{C}\right)\end{array}$ & $\begin{array}{c}\Delta H_{\mathbf{c}} \\
(\mathrm{J} / \mathbf{g})\end{array}$ \\
\hline PA-11 & 187.5 & 43.5 & 159.3 & 48.4 & 186.4 & $\mathbf{4 0 . 7}$ & 159.0 & 48.1 \\
\hline PA-11/Na-MMt & 186.3 & 50.1 & 159.3 & 51.3 & 186.1 & 39.5 & 159.1 & 54.5 \\
\hline PA-11/OM-MMt & 188.0 & 50.5 & 160.5 & 52.0 & 187.0 & $\mathbf{4 0 . 0}$ & 160.1 & 54.1 \\
\hline PA-11/AO-OM-MMt & 188.2 & 47.7 & 160.3 & 51.0 & 187.3 & $\mathbf{3 8 . 6}$ & 160.0 & 52.2 \\
\hline PA-11/OM-MMt/Irg & 188.1 & 48.6 & 160.2 & 50.7 & 187.0 & $\mathbf{4 0 . 1}$ & 159.5 & 52.8 \\
\hline
\end{tabular}

\subsection{Thermo-oxidative Stability of PA-11- Nanocomposites}

It is well known that hydrocarbon polymer-based nanocomposites are susceptible to thermo-oxidative degradation during melt processing as well as in the course of their lifetime, so it is a common practice to assess their stability by performing accelerated degradation tests at high temperature. Rheology is also frequently used for this purpose as it is particularly sensitive to molecular architecture, and is used to infer the occurrence of degradation. To investigate the thermo-degradation of the PA-11-PNC during melt processing, rheological analysis was performed and the complex viscosity, $\eta^{*}$, and moduli G' and G" are shown in Fig. 13. As can be expected, the viscosity values of all the nanocomposites are significantly higher than those of neat PA-11 in all the frequency range examined due to the well exercised filler effect. However, there are differences in the viscosities of the different nanocomposites, for example the presence of the lab-made OMMMt leads to a slight increase in viscosity values, albeit much lower than that of the PA-11/Na-MMt sample. This is most likely due to the well-known Hoffman degradation of the ammonium-based OM as a consequence 
of thermo-oxidative degradation by PA-11/OM-MMt nanocomposite containing the lab-made OM-clay during processing [10-24]. However, for samples containing the hindered phenol antioxidants, they show higher viscosity values with the effect being more pronounced for the PA-11/AO-OM-MMt-containing PNC. The G' and G" curves for the samples containing OM-MMt and particularly the sample with AO-OM-MMt examined at low frequency showed a well noticeable yield stress of G' vs $\omega$ suggesting a good filler dispersion and formation of threshold filler network (see Fig. 13 b).

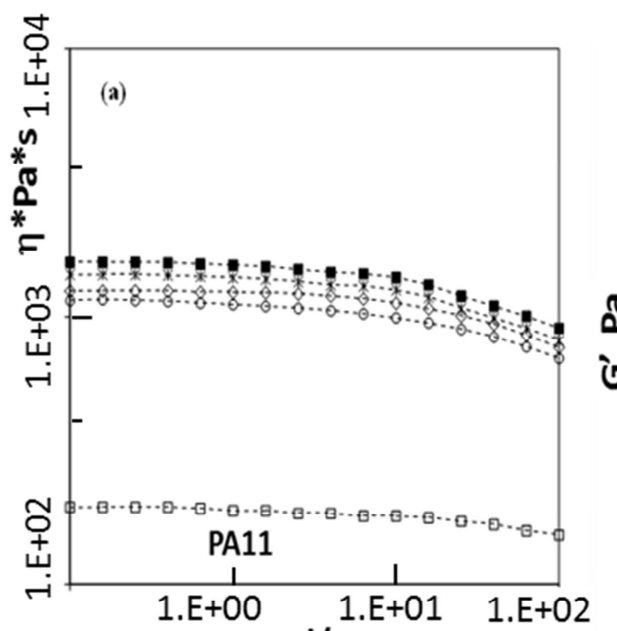

$\omega, \mathrm{rad} / \mathrm{s}$

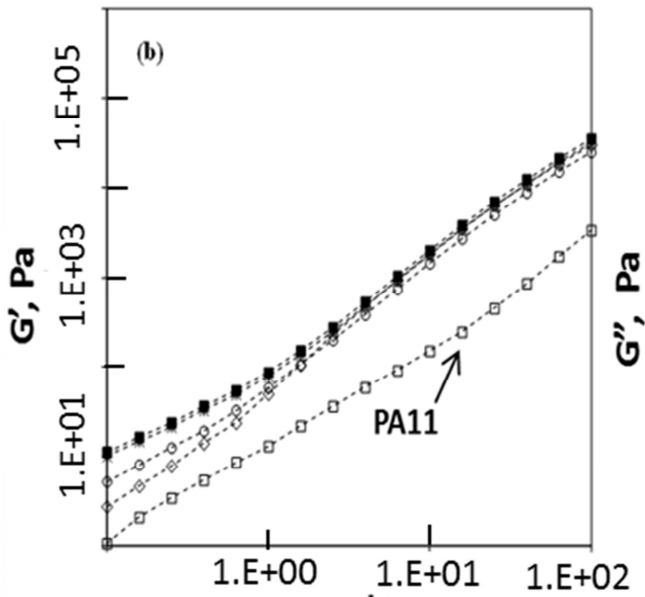

$\omega, \mathrm{rad} / \mathrm{s}$

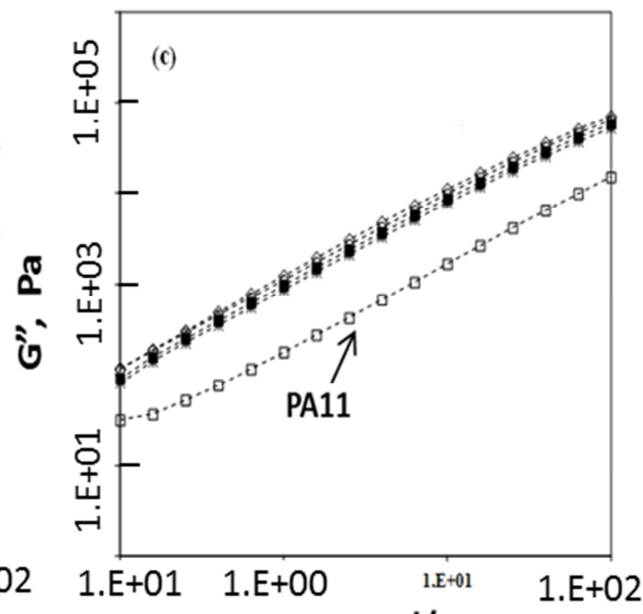

$\omega, \mathrm{rad} / \mathrm{s}$

口PA11; $\diamond$ PA11/NaMMt; OPA11/OM-MMt; - PA11/AO-OM/MMt; * PA11/OM-MMT/Irg

\section{Figure 13}

The long-term thermo-oxidative resistance of both neat PA-11 and the PA-11- PNC samples were investigated by studying the thermal ageing of thin films at $170^{\circ} \mathrm{C}$ in an air oven followed by FTIR analysis. It is well known that hydrolysis is the predominant degradation pathway for polyamides in the presence of humidity, while random chain scission reactions and the formation of oxygen containing groups (e.g. carbonyl and/or carboxyl groups) are the main degradation routes during thermo- or photo- oxidation [40-43]. Based on the rate of growth of the carbonyl groups, the neat PA-11 matrix is shown to undergoe fast thermo-oxidation, Fig. 14. The nanocomposite sample containing the unstabilised lab-made organic modifier (OM-MMt clay) shows a faster rate of thermal oxidation than both the PA-11 matrix and nanocomposites containing unmodified NaMMt. On the other hand, the presence of an antioxidant (either 'free', added to the composition or chemically reacted onto the organo-modifier) gives rise to significant increases in the thermo-oxidative resistance of the nanocomposites samples (see Fig 14). The beneficial effect is however, more pronounced in the case of the chemically-reacted antioxidant (PA-11/AO-OM-MMt) compared to the stabilised sample containing the added ('free' unreacted) commercial hindered phenol antioxidant, Irganox 1076 (PA11/OM-MMt//rg), in spite of the fact that the latter was prepared with twice the amount of the antioxidant. i.e. Irg1076 at $1 \mathrm{wt} \%$, as that present in the reacted antioxidant (AO-OM) with a concentration of about $0.5 \mathrm{wt} \%$ (calculated from thermogravimetry, TG). 
The lower thermoxidative stability observed in the presence of the added ('free' unreacted) antioxidant (sample PA11/OM-MMT/Irg) is most likely due to the partial loss of the protective action of the antioxidant due to both an easier depletion (than if chemically reacted) and a partial deactivation of the hindered phenol moiety by the organo-modified clay for reasons that have been reported previously for clay-filled polymer nanocomposites [17] A further evidence of the higher thermo-oxidative resistance achieved by the chemically reacted antioxidant (PA-11/AO-OM-MMt) can be seen from a visual inspection of the samples at the end of the thermal exposure experiment. Figure 14 shows that the neat PA-11 appears to have a non-uniform yellow discolouration due to the formation of UV/vis active chromophores typically formed during aliphatic polyamides oxidation [41]. Similar observations were made with all the other samples, although the overall discolouration appears to be little more uniform, with the exception of the PA11/AO-OM-MMt which shows a partial yellowing only. This supports further the overall higher long-term thermal protection observed for the PA-11 nanocomposites containing this new (reacted) AO-containing organo-modified clay.
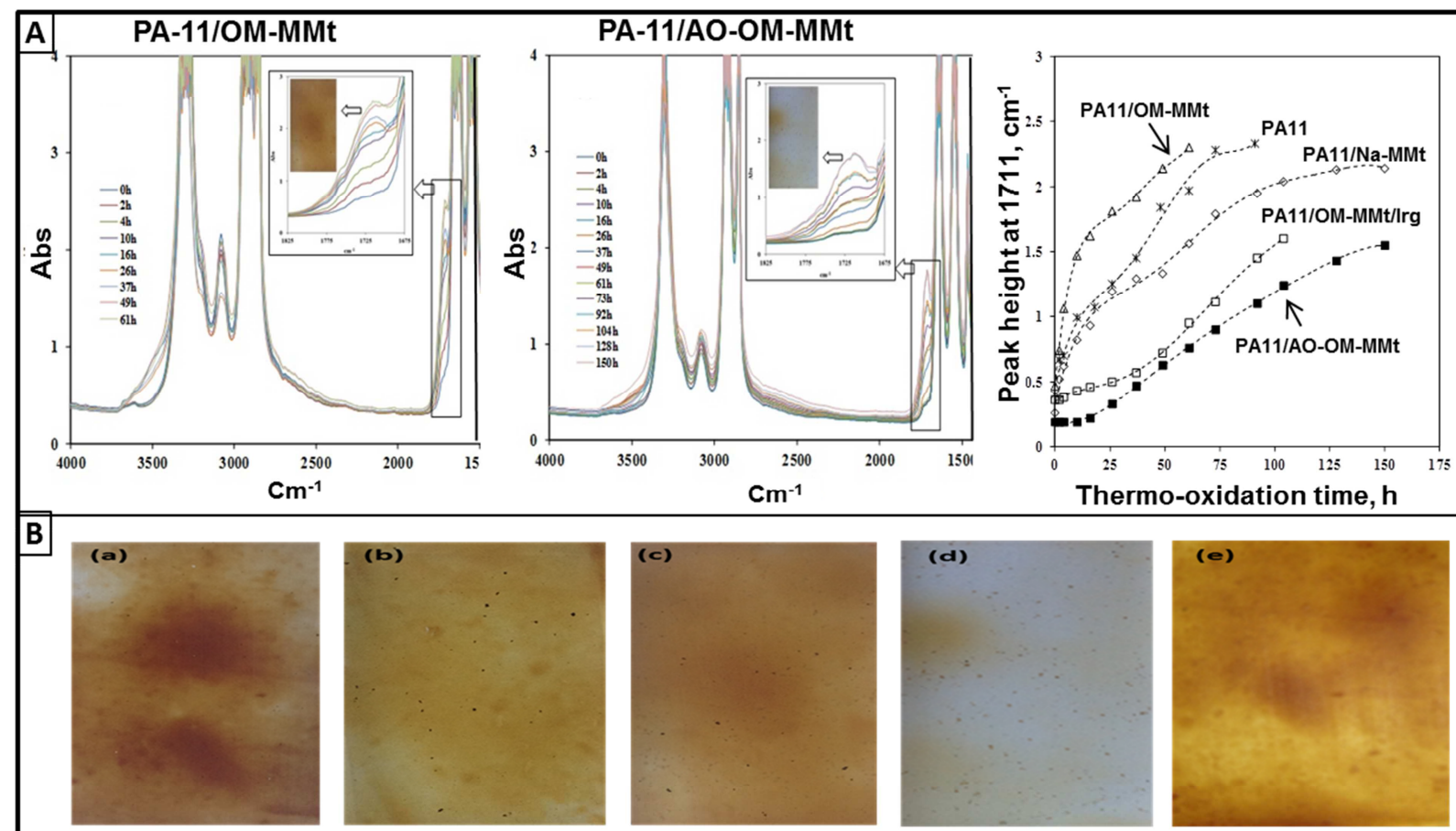

PA11

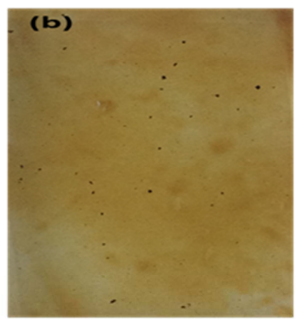

PA11/Na-MMt

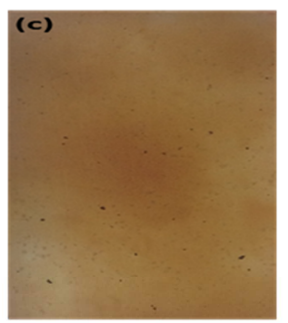

PA11/OM-MMt
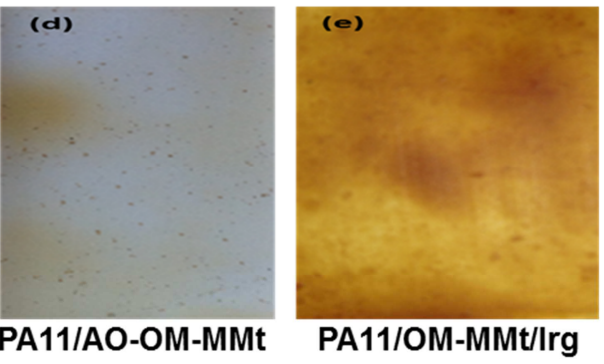

Figure 14

\section{Conclusions}

Maintaining the long-term performance during the lifetime of hydrocarbon polymer-clay based nanocomposites is critical for their successful end-use applications. In the last two decades, huge attempts were made to finding solutions to the issues of low thermal and photo-oxidative stability of these nanocomposites but none of these routes have been completely satisfactory. 
In this work, a new novel approach has been investigated whereby an antioxidant function was reacted chemically with the quaternary ammonium organic modifier before exchanging with the Na-MMt clay followed by the production of the PA-11-nanocomposites. The results obtained show excellent dispersion and exfoliation in the in-situ stabilised/modified clay nanocomposite (PA-11/AO-OM-MMt) with a very good overall processing and long-term thermo-oxidative stability which was shown to be better than that of a similar nanocomposite sample but where the antioxidant was conventionally added (and in excess) to the formulation rather than being chemically reacted (PA-11/OM-MMt//rg). The work described in this paper has focused only on PA-11-nanocomposites but future publications will focus on other polymer matrices. This AO-containing OM-based clay system, therefore, may offer a new and highly effective approach for the stabilisation of hydrocarbon-based polymer-clay nanocomposites.

Acknowledgments: This work was financially supported by EU Marie Curie Action-PEOPLE 2011-IEF, (N:300302), Project: NANOSTAB-GB - Novel Nano-Stabilisation for Green Bioplastic Nanocomposites. Our thanks are also due to Dr K Doudin for his help with the NMR, to Dr H H Sheena for general discussions, and to Professor Gambarotti for the chemical modification of the organo-modifier and for useful discussion.

\section{REFERENCES}

[1] Gilman JW. Appl Clay Sci 1999;15:31.

[2] Beyer G. Plast Addit Compound 2002;4:22.

[3] Yano K, Usuki A, Okada A, Kurauchi T, Kamigaito O. J Polym Sci Polym Chem 1993;31:2493.

[4] Dintcheva NTz, Filippone G, La Mantia FP, Acierno D, Polym Degrad Stab 2010;95:527.

[5] Pinnavaia TJ. Polymer clay nanocomposites. New York: Wiley; 2000.

[6] Alexandre M, Dubois P. Mater Sci Eng 2000;28:1.

[7] Ray SS, Okamoto M. Prog Polym Sci 2003;28:1539.

[8] Pavlidou S, Papaspyrides CD. Prog Polym Sci 2008;33:1119.

[9] Paul DR, Robeson LM. Polymer 2008;49:3187.

[10] Xie W, Gao Z, Pan W-P, Hunter D, Singh A, Vaia R. Chem Mater 2001;13:2979.

[11] Xie W, Gao Z, Lin K, Pan W-P, Vaia R, Hunter D, Simgh A. Thermochim Acta 2001;367:339-50.

[12] Tidjani A, Wilkie CA, Polym Degrad Stab 2001;74:33-7.

[13] Xie W, Xie R, Pan W-P, Hunter D, Koene B, Tan L-S, Vaia R. Chem Mater 2002;14:4837.

[14] Qin H, Zhao C, Zhang Z, Chen G, Yang M. Polym Degrad Stab 2003;81:497-500.

[15] Fornes TD, Yoon PJ, . Paul DR. Polymer 2003:44:7545-56.

[16] Mailhot B, Morlat S, Gardette JL, Boucard S, Duchet J, Gerard JF. Polym Degrad Stab 2003;82:163-7.

[17] Morlat S, Mailhot B, Gonzalez D, Gardette JL. Chem Mater 2004;16:377-83.

[18] Pandey JK, Reddy KR, Kumar AP, Singh RP. Polym Degrad Stab 2005;88:234.

[19] Shah RK, Paul DR. Polymer 2006;47:4075-84.

[20] La Mantia FP, Dintcheva NTz, Malatesta V, Pagani F. Polym Degrad Stab 2006;91:3208-13.

[21] Qin H, Zhang Z, Feng M, Gong F, Zhang S, Yang M, J Polym Sci Part B: Polym Phys 2004;42:300612.

[22] Bocchini S, Morlat-Therias S, Gardette JL, Camino G. Polym Degrad Stab 2007;92:1847-56.

[23] Botta L, Dintcheva NTz, La Mantia FP. Polym Degrad Stab 2009;94:712.

[24] Dintcheva NTz, Al-Malaika S, La Mantia FP. 2009;94:1571.

[25] Pfaendner R. Polym Degrad Stab 2010;95:369.

[26] Al-Malaika S., Sheena H, Fischer D, Masarati E. Polym Degrad Stab 2013;98:2400

[27] Dintcheva NTz, La Mantia FP, Malatesta V. ex-Press Polym Letter 2011;5:923. 
[28] Chowa WS, Lima SR. Polym-Plast Technol Eng 2013;52;626.

[29] Filippi S, Dintcheva NTz, Scaffaro R, La Mantia FP, Polacco G, Magagnini PL. 2009;49:1187.

[30] Calderon JU, Lennox B, Kamal MR. Appl Clay Sci 2008:40:90.

[31] Yei DR, Kuo SW, Su YC, Chang FC. Polymer 2004;45:2633.

[32] Saikai BJ, Parthasarathy G. J Mod Phys 2010;1:206.

[33] Oliveira MJ, Botelho G. Polym Degrad Stab 2008:93:139

[34] Filippone G, Carroccio SC, Curcuruto G, Passaglia E, Gambarotti C, Dintcheva NTz. Polymer 2015;73:102

[35] Filippi S, Paci M, Polacco G, Dintcheva NTz, Magagnini PL. Polym Degrad Stab 2011;96:823.

[36] Zhang G, Li Y, Yan D. J Polym Sci: Part B 2004;42:253.

[37] Zhang Q, Yu M, Fu Q. Polym Int 2004;53:1941.

[38] Fornes TD, Paul DR. Polymer 2003;44:3945.

[39] Kojima Y, Usuki A, KawasumiM, Okada A, Kurauchi T, Kamigaito Q, Kaji A, J. Polym Sci: Part B: Polym Phys 1994;32:625

[40] Rabek JF. Photodegradation of polymers. Berlin/Heidelberg:Springer; 1996.

[41] Thanki PN, Singh RP. Polymer 1998;39:6363.

[42] Levchik SV, Weil ED, Lewin M. Polym Int 1999;48:532.

[43] Oliveira MJ, Botelho G. Polym Degrad Stab 2008;93:139.

\section{CAPTION OF FIGURES}

Figure 1. FTIR spectral regions for $\mathrm{AO}-\mathrm{OM}$ and $\mathrm{OM}$ in $\mathrm{KBr}(\mathrm{a})$ and the corresponding deconvoluted peaks $(\mathrm{b}-\mathrm{c})$

Figure 2. ${ }^{1} \mathrm{H}$ NMR spectra of the synthesised AO-OM and of the corresponding $\mathrm{OM}$

Figure 3. Mass spectrum of AO-OM

Figure 4. FTIR spectra in the range $1900-1200 \mathrm{~cm}^{-1}$ for the different clays in $\mathrm{KBr}(\mathbf{a})$, the deconvoluted peaks for AO-OM-MMT(b) and the lab-made OM-MMT (c)

Figure 5. FTIR spectra in the range 3800 to $2700 \mathrm{~cm}^{-1}$ for the different clays in $\mathrm{KBr}(\mathbf{a})$, the deconvoluted peaks for AO-OM-MMT(b) and the lab-made OM-MMT (c)

Figure 6. XRD patterns of Na-MMT, OM-MMt and AO-OM-MMt clays (The clay curves have been shifted on the $y$-axis for clarity).

Figure 7. TG curves of Na-MMT, OM-MMt and AO-OM-MMt clays

Figure 8. FTIR spectra in $\mathrm{KBr}$ with ageing time of the different clays (Na-MMt, OM-MMt and AO-OM-MMt)

Figure 9. Torque changes during melt processing of the different PA11-nanocomposites

Figure 10. XRD patterns of the different PA-11-nanocomposites

Figure 11. SEM and TEM micrographs of PA-11/Na-MMt (a-c), PA-11/OM-MMt (d-f), and PA-11/AO-OM-MMt (j-1) at different magnifications

Figure 12. DSC curves (second heating scan) of neat PA-11 and its nanocomposites

Figure 13. Viscosity curves (a), modulii $G^{\prime}(\mathbf{b})$ and $G^{\prime \prime}(\mathbf{c})$ of neat PA-11 and its nanocomposites.

Figure 14. Changes in FTIR spectra with ageing time (at $170^{\circ} \mathrm{C}$ ) of PA-11/OM-MMt and PA-11/AO-OM-MMt, and growth in the carbonyl peak height at $1711 \mathrm{~cm}^{-1}$ for different PA11-nanocomposites is also shown (A) with visual images of different nanocomposite films at maximum ageing time of $150 \mathrm{~h}$ displayed in (B).

\section{CAPTION OF TABLES}

Table 1. Commercial clay nanofillers used and their characteristics (manufacturer's data)

Table 2. Melting $\left(\mathrm{T}_{\mathrm{m}}{ }^{\circ} \mathrm{C}\right)$ and crystallization $\left(\mathrm{T}_{\mathrm{c}}{ }^{\circ} \mathrm{C}\right)$ temperatures, and melting $\left(\Delta \mathrm{H}_{\mathrm{m}}\right)$ and crystallisation $\left(\Delta \mathrm{H}_{c}\right)$ enthalpies (corrected for the clay content) for neat PA-11 and its nanocomposites (from DSC measurements) 


\section{Supplementary Information}

\section{S1: Characterization of Irganox acid ( $r-A O)$}

Characterisation of Irganox acid (r-AO) was carried out using NMR, FTIR and MS spectroscopy.

A comparison of the ${ }^{1} \mathrm{H}-\mathrm{NMR}$ spectrum of the r-AO with that of the starting material (Irganox 1076) shows that in the case of the Irganox acid ( $\mathrm{r}-\mathrm{AO})$ the chemical shifts of the methylene and methyl groups of the stearyl moiety in the starting material (Irg 1076: signals C, F, J, K, L) have clearly disappeared, see Figure S1A,B.

\section{A: Irg Acid ('H-NMR)}

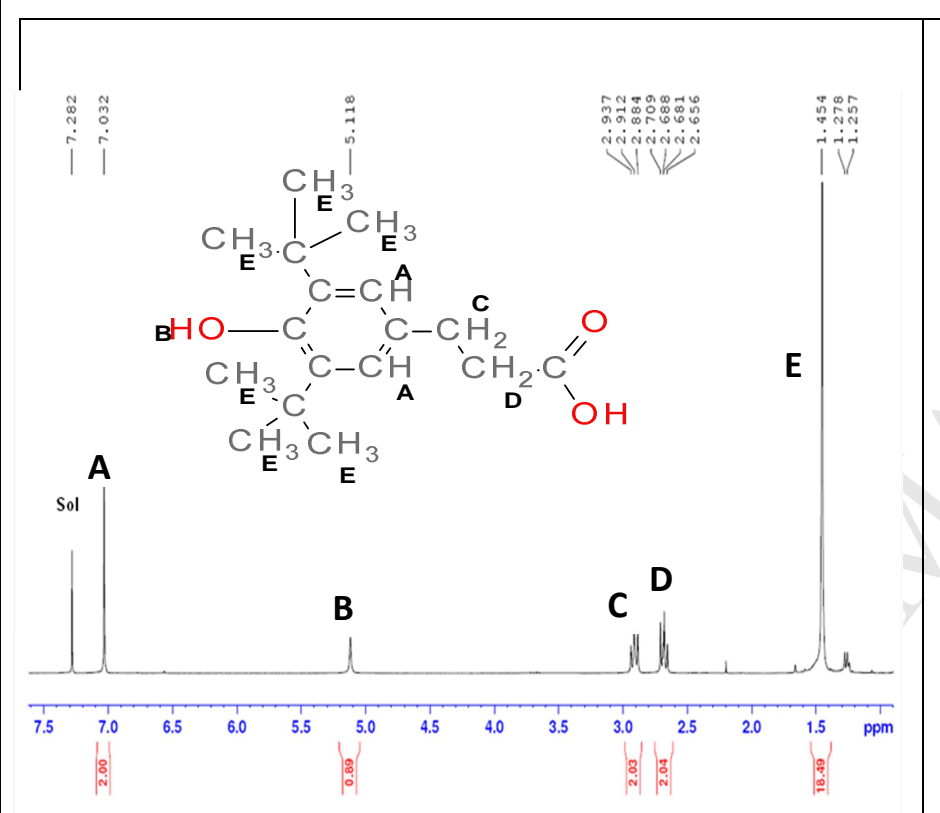

B: $\operatorname{Irg} 1076$ ('H-NMR)

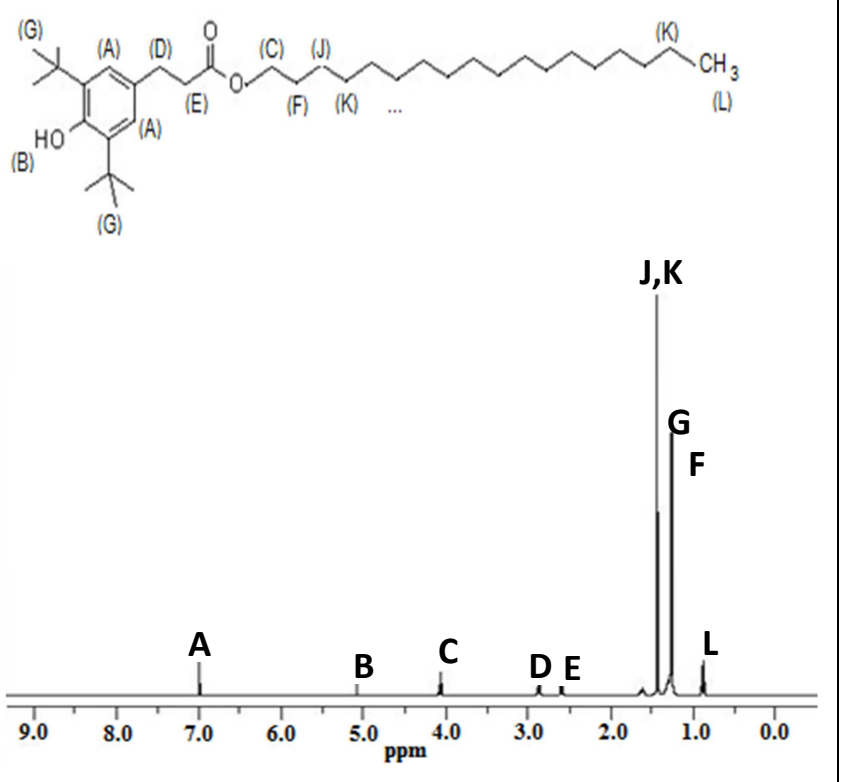

\section{D: Mass spectrum of Irg Acid}

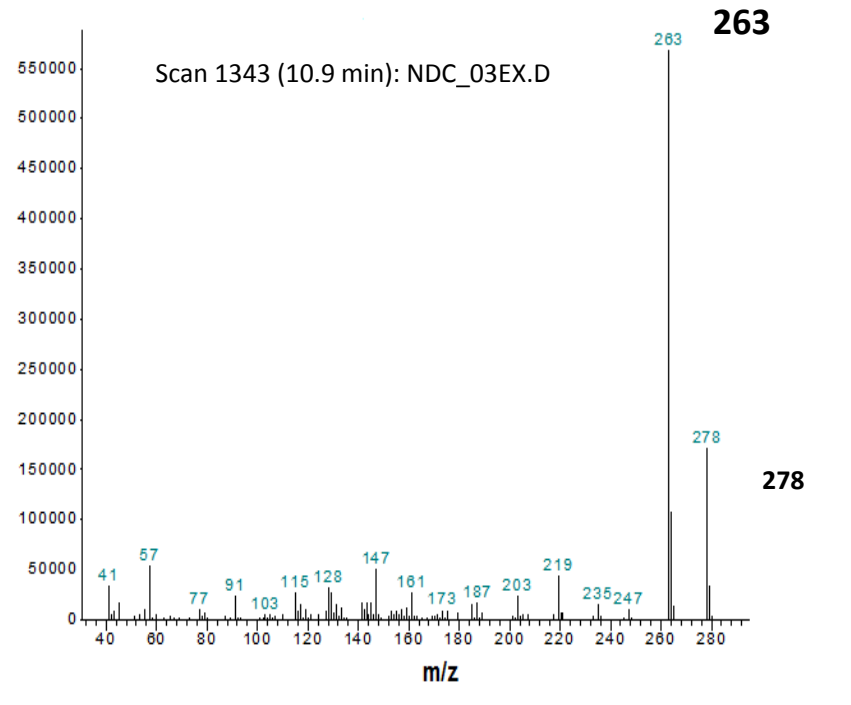

Figure S1. 1H NMR spectra of Irganox acid (A) and Irganox 1076 (B); FTIR of Irganox acid and Irganox 1076 (C); mass spectrum of Irganox acid (D) 
The FTIR spectrum in the carbonyl region confirms further the formation of the acid and shows a clear shift in the carbonyl absorption from an ester-type (1734 $\mathrm{cm}^{-1}$ in Irganox 1076) to an acid-type (1707 $\mathrm{cm}^{-1}$ in r-AO), Fig. 1C. The infrared sperctrum of Irganox acid also shows the appearance of two peaks in the C-O stretching (1320-1210) and $\mathrm{O}-\mathrm{H}$ bending (1440-1395 $\left.\mathrm{cm}^{-1}\right)$ regions. The molecular mass of Irganox acid is $262 \mathrm{~g} / \mathrm{mol}$, and its mass spectrum confirms that the molecular ion peak has a mass of 263, Fig 1D (compared to a mass of $531 \mathrm{~g} / \mathrm{mol}$ for Irganox 1076).

\section{S2: Comparison between lab-made OM-MMt and commercial Cloisite $₫ 30 B$}

No significant differences between lab-made OM-MMt and commercial Coisite $₫ 30 \mathrm{~B}$ was detected based on their FTIR and TGA analysis, see Figure S2 a-b. Both samples also follow the same degradation pathways as can be seen from the changes in their FTIR spectra with thermo-oxidation time.
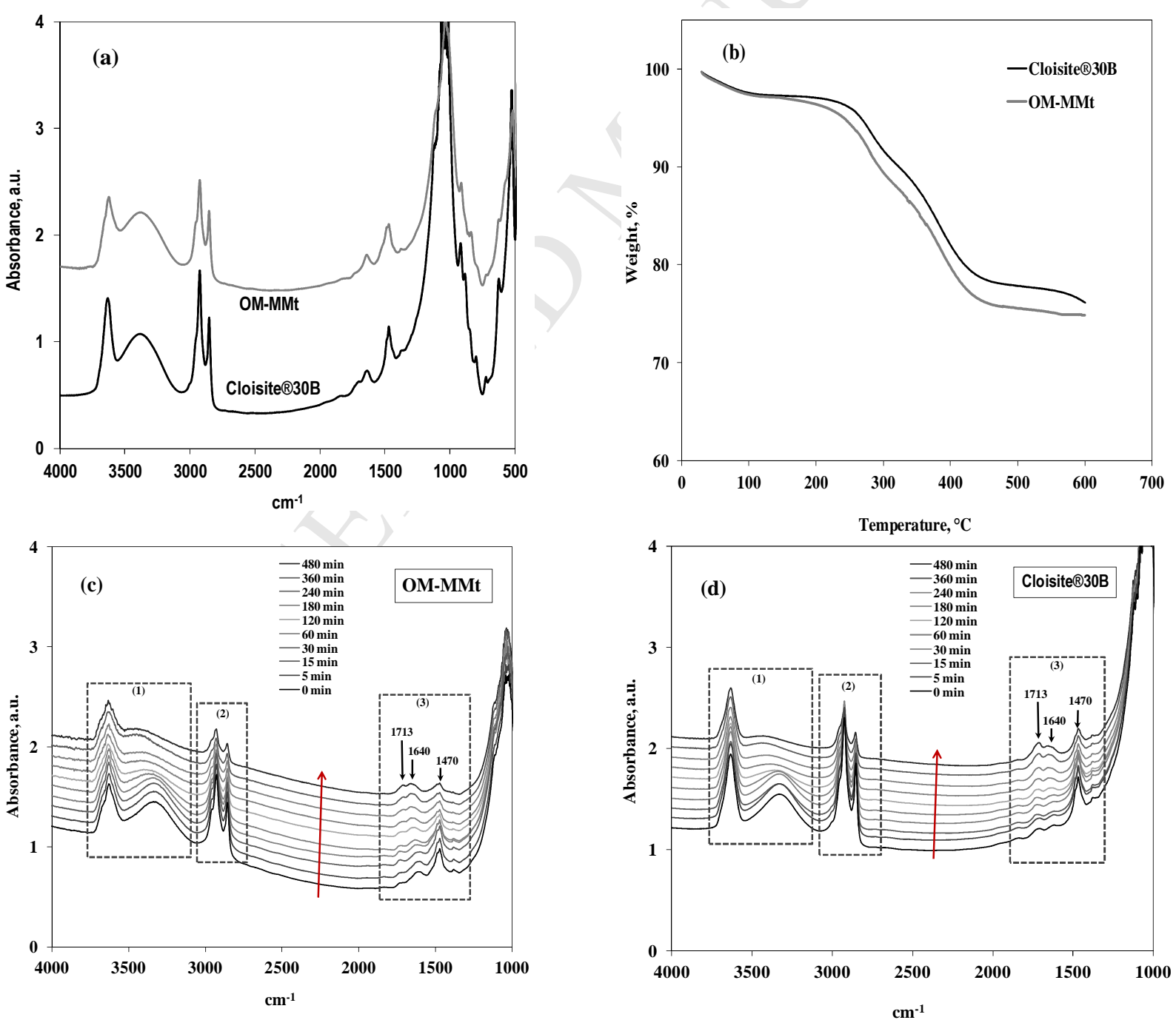

Figure S2. FTIR spectra (a) and TGA (b) of lab-made OM-MMt and commercial Cloisite $₫ 30 B$; FTIR spectra at different thermo-oxidation times of OM-MMt (c) and Cloisite $₫ 30 B(d)$ 